\title{
Performance of $T$ shape barriers with top surface covered with absorptive Quadratic Residue Diffusers
}

\author{
M. R. Monazzam and Y. W. Lam \\ Acoustics Research Centre, School of Computing, Science and Engineering \\ University of Salford, Salford, M5 4WT, UK
}

\begin{abstract}
A previous paper [Applied Acoustics 66, pp.709-730, 2005] has shown that adding a quadratic residue diffuser ( $Q R D)$ to the top of a T-shape barrier can provide better barrier performance than an equivalent purely absorptive barrier. In here, we extend the study to look at the performance when a QRD is made absorptive. This paper presents an investigation on the acoustic performance of a few welled-diffusers with different absorption ability on top of a T-shape noise barrier. The absorption properties of the diffusers are modified with different sequences, by filling the wells with fiberglass, by covering the well entrance with wire meshes, and by putting perforated sheet either on the top surface or inside the wells. A 2D Boundary Element Method (BEM) is used to calculate the barrier insertion loss. The numerical and experimental results on diffuser barriers with rigid and absorptive covers are compared. Among the tested models the best method of treating diffuser barriers with absorbent agents in the QRD is found to be a perorated sheet on top or inside the diffuser wells. It is found that increasing the absorption ability of $Q R D$ by fiberglass or high resistance wire meshes has negative effect on the efficiency of a QRD barrier. It is shown that, if the increase in absorption destroys the effect of resonance in wells, it will also have negative effect on the insertion loss performance of the QRD edge barrier.
\end{abstract}

\section{Introduction}

Traffic noise barriers are the most common solution for controlling unwanted noise from expressways and other outdoor noise sources. A number of methods have been suggested 
to improve their effectiveness without increasing their height. The barrier edge is known to act as an imaginary source for a diffracted field at the back of the barrier [1]. Control of sound pressure at the barrier's edges therefore reduces this imaginary source and decreases the diffracted field at the rear of the barrier. Previous works found that employing a weakly absorbing upper surface to a T-shape profile barrier could not improve the efficiency of the barrier significantly, while more strongly absorbent surface gave increasingly higher improvements [2]. Covering a T- shape barrier with a layer of mineral wool type absorbent material (flow resistivity $\sigma=20000 \mathrm{Nsm}^{-4}$ and thickness $\mathrm{T}=0.1 \mathrm{~m}$ ) could improve the performance of a single rigid barrier by $2.5 \mathrm{~dB}$ according to D.C. Hothersall et al [3].

In order to give more suppression of the sound pressure, Fujiwara [4] introduced a barrier with an "acoustically soft surface", where sound pressure is much less than that of an absorptive surface. Although improvement over a wide frequency range wasn't obtained, the efficiency of the "soft" barrier increased by more than $10 \mathrm{~dB}$ in the frequency range where the surface pressure was minimised. It is also worth noting that, providing such a soft surface with wide range frequency effect is very difficult. Fujiwara tried to introduce a practical "Cylindrical edge barrier" for practical use [1]

Utilising quadratic residue diffuser (QRD) on different barrier profiles is also investigated by the authors, where the best shape for using the device was found to be a $\mathrm{T}$-shape profile with a $400 \mathrm{~Hz}$ design frequency [5]. It was found that a $400 \mathrm{~Hz}$ frequency designed T-shape QRD barrier could increase the efficiency beyond its equivalent absorbent $\mathrm{T}$-shape barrier by up to $3.5 \mathrm{~dB}$ (A) on a rigid ground. The highest improvement was achieved in the far field at heights between 6 to $8 \mathrm{~m}$ above the ground. Although QRD is well known as a diffuser that spreads sound in many directions with very low loss in energy, there are also a few studies showing that they can also work as an absorbent device. Commins, Auletta and Suner [6] experimentally investigated the absorption characteristics of a Schroeder diffuser. They showed that by slopping the bottom of the diffuser wells, the absorption could be reduced. K. Fujiwara [7] in an experimental investigation found a high absorption coefficient on a Schroeder diffuser 
particularly at frequencies lower than the design frequency. H. Kuttruff [8] later confirmed the high absorption of the diffuser at low frequencies. The reason behind this unexpected absorption was thought to be strong interaction between elements, that each of them being tuned to a different resonant frequency. More investigation both experimental and numerical on the reasons for the very high absorption ability of Schroeder diffuser (QRD) was done by Fujiwara [9]. At the same time an analytical solution for the absorption coefficient of a welled diffuser was also introduced by F.P. Mechel [10].

$\mathrm{T}$. Wu et al [11] introduced an optimised QRD absorber and they verified the effectiveness of a resistive layer at the well opening for increased absorption. They obtained very high absorption performance from a Schroeder diffuser. They also introduced a type of profiled absorber in another investigation [12] which could extend the absorption at low frequencies whilst preserving the absorption at mid frequencies. However there is yet no application of QRD as an absorbent in environmental noise control.

This paper investigates the effect of a new set of barrier types using various welled diffusers with different absorption properties on the top surface of a T-shape barrier. In this report the performance of noise barriers with welled diffusers and QRD profiles with either wire mesh, perforated sheet or fibreglass, is calculated using a two-dimensional boundary element method. Insertion loss spectra at 1/15-octave centre frequencies are calculated. The results are compared with absorptive as well as a selected plain QRD top surface $\mathrm{T}$-shape barrier on rigid ground to show when it is efficient to add absorption to a QRD in barrier applications.

\section{Schroeder Diffusers}

Depth sequence diffusers were introduced by Schroeder in the $1970[13,14]$. The most common of them is the Quadratic Residue Diffuser (QRD). They are periodic surface structures with rigid construction that are intended to reduce specular sound reflection by scattering the incident sound energy in to a wide range of directions. The elements of the 
construction are wells with the same width separated by thin fins. In this case the well depth sequence for a QRD is determined by the quadratic residue sequence, which is a mathematical sequence based on a prime number, $\mathrm{N}$. The $n$th term in the sequence is given by $n^{2} \bmod \mathrm{N}$. Ideally a QRD should produce a uniform scattered field at its design frequency. Primitive root sequence is one more example of a mathematical sequence providing another kind of Schroeder diffuser known as primitive root diffuser (PRD).

\section{Absorption by Schroeder Diffusers}

Schroeder diffuser can absorb sound energy mainly because of (i) high energy flows from wells in resonance to wells out of resonance, and (ii) quarter wave resonant absorption in the wells, particularly in narrow wells. In an investigation Fujiwara [9] looked at the possible reasons behind the absorption ability of Schroeder diffusers. Quadratic residue sequence diffuser was found to have lower absorption ability than random and regular sequence diffuser over the entire tested frequency range [Figure 6 of Ref 9]. Moreover, in his numerical prediction, which had fairly good agreement with experimental result, it was shown that $\mathrm{QRD}$ with higher prime number $(\mathrm{N}=11)$ got more absorption coefficient than one with $\mathrm{N}=7$. Finally he concluded that even if the inner surface of a $Q R D$ is ideally smooth the absorption coefficient was as high as 0.3 in the frequency range below the design frequency. Furthermore absorption coefficients were found to be very frequency dependent and very sharp absorption peaks were observed at the resonance frequencies of the coupled wells.

An optimised well sequence was shown to have better absorption ability than a QRD and random structure diffusers according to T. Wu et [11]. Both $\mathrm{H}$. Kuttruff [8] and T. Wu et [11] tried to show the importance of the surface covering of a QRD in increasing the absorption ability of the device. There is energy flow between wells of the diffuser promoted by pressure gradients, which are caused by wells being in resonance and wells not in resonance. Therefore high particle velocity around the front face of a Schroeder 
diffuser occurs, and a resistance cloth covering will cause excess absorption as might be expected if resistive material is placed in a region of high particle energy flow.

$\mathrm{T}$. Wu et [11] showed the dramatic effects of covering a QRD with wire mesh, which made huge increases in the absorption coefficient of constant slit diffusers, QR diffusers, and an optimised QR diffuser. In another investigation a new idea of improving absorption coefficient of QR diffuser was raised by $\mathrm{T}$. Wu et [12]. It was proved both experimentally and theoretically that not only covering the surface of QR diffusers by a perforated sheet is effective in increasing the absorption ability of the structure, but also having a variably positioned perforated sheet in the well introduces extra degree of freedom that produces a good broad band absorption coefficient for QR diffusers. These means of increasing the absorption property of a QRD, and their effect on barrier performance will be tested here.

\section{Numerical Modelling Method}

A noise barrier of infinite length lies on the plane, and it is assumed that the acoustical properties and the cross-section shape of the noise barrier do not vary across its length. The problem is therefore two-dimensional, with the z-axis parallel to the barrier length. The geometrical and acoustical variables are constant in the z-direction. The barrier surface is assumed to be locally reacting with specific surface admittance. The Helmholtz wave equation is solved by the boundary integral equation at a single frequency using the boundary element method [5].

The welled diffuser is represented by a box with the top surface having an admittance distribution as given by the simple phase changes due to plane wave propagation within the diffuser's wells. This assumption has been validated on a QRD barrier by the authors

[5]. It was shown that the result of box approximation method has very good agreement up to just below the upper cut off frequency. In order to validate the box approximation 
method for the QRD barriers within the interested frequency range of this investigation, the results of an experimental study will be reported later in Section 7.

The interference between the source and its ground image was avoided by locating the sound source on a rigid ground. To represent typical distances of high speed traffic on single and double carriageway roads adjacent to roadside barriers where rolling noise predominates, the distance from the source to the centre line of the barrier is chosen at 5 $\mathrm{m}$. The sound pressure is predicted at 1/15-octave centre frequencies between 50 and $4000 \mathrm{~Hz}$ at different receiver locations. The insertion loss at each frequency is calculated by:

$I L=20 \log _{10}\left|\frac{p_{g}}{p_{b}}\right| \quad d B$

where $p_{g}$ is the pressure at the receiver with only the flat ground present, and $p_{b}$ is the pressure with both the ground and barrier present.

\section{Absorbent effect}

\subsection{Fibrous material}

The empirical formulae of Delany and Bazley [15] are used for the calculation of the characteristic impedance $Z_{c h}$ and propagation constant $\gamma$ of fibrous materials.

$z_{c h}=\rho c\left(R_{g}+j X_{g}\right)$,

where:

$R_{g}=1+9.08\left(\frac{\sigma}{1000 f}\right)^{0.75}, X_{g}=-11.9\left(\frac{\sigma}{1000 f}\right)^{0.73}$

$\sigma$ is the flow resistivity. The propagation constant is:

$\gamma=a+j \beta$,

where: 
$a=\frac{2 \pi f}{c} 10.3\left(\frac{\sigma}{1000 f}\right)^{0.59}, \beta=\frac{2 \pi f}{c}\left\{1+1.08\left(\frac{\sigma}{1000 f}\right)^{0.7}\right\}$,

The flow resistivity of the fibrous material is chosen to be a typical 20000 Rayls(MKS), which is different from Ref.[16]'s assumption of an ideal specific normal surface impedance of 1 for a perfectly absorbing surface. Using a typical fibrous cloth is considered to be more realistic than assuming the ideal absorptive condition. The absorption coefficient is then calculated by

$\alpha\left(\theta_{e}\right)=1-\left|\frac{Z_{c h} \cos \left(\theta_{e}\right)-1}{Z_{c h} \cos \left(\theta_{e}\right)+1}\right|^{2}$

where $\theta_{e}$ is the angle of incident

\subsection{Diffusers}

\section{a. Impedance of a profiled absorber}

i. The impedance at well entrance of a Schroeder diffuser

If the surfaces of the wells are rigid and it is sufficiently wide the viscous and thermal losses are generally small. However, for completeness, the method introduced by $\mathrm{T}$. Wu et al [11] is used to calculate the normalised specific impedance of the wells. In this method the inward impedance of the surface of a well with depth $l_{n}$ is calculated by:

$z=-j \rho_{e} c \frac{k}{k_{t}} \cot \left(k_{t} l_{n}\right)$

where $k_{t}$ is the propagation number, $k$ is the wave number and $\rho_{e}$ is the effective density of air in the well. More details on this method could be found in Reference [11].

\section{ii. The impedance of a perforated sheet}

The ability of a perforated panel to produce high absorption at low frequencies in combination with a cavity is well known. Two assumptions are usually made in 
calculating their impedance, including a) no motion in the plate exists when sound flows through the holes, and b) the radius of hole is larger than the boundary layer thickness. The method presented in [12] is used to calculate the specific acoustic impedance of the perforated sheet. In this method the impedance at the opening of a well is derived by the multilayer transfer matrix as:

$z=\frac{\rho c z_{1} \operatorname{coth}\left(j k_{t} l_{n 1}\right)+(\rho c)^{2}}{z_{1}+\rho c \operatorname{coth}\left(j k_{t} l_{n 1}\right)}$,

where $k_{t}$ is the propagation number, $l_{n 1}$ is the depth from the sheet to the well bottom, and $z_{1}$ is the backing impedance of the cavity. More descriptions on this method could be found in Reference [12].

If the cavity is backed by another perforated cavity, then $z_{1}$ can be calculated by;

$z_{1}=r_{m}+j\left(\omega m-\rho c \cot \left(k_{t}\left(l_{n}-l_{n 1}\right)\right)\right)$

where $r_{m}$ and $\mathrm{m}$ are respectively the resistance and effective mass of the dividing perforated sheet, and $l_{n}$ is the depth of the bottom backing cavity. The perforated plate is fixed at $l_{n 1}$.

\section{iii. The impedance at entrance of a well having resistive layer}

The impedance at the entrance of a well covered by a resistive material can similarly be derived by multilayer transfer matrix as $[11,12]$

$z=\frac{\rho c z_{1} \operatorname{coth}\left(j k_{t} l_{n 1}\right)+(\rho c)^{2}}{z_{1}+\rho c \operatorname{coth}\left(j k_{t} l_{n 1}\right)}+r$

Resistivity of the material is given by $r$ in this equation. The other elements of the equation were as defined in Equations 8 and 9.

\section{b. Absorption by a profiled absorber}

The absorption coefficients at different incident angles for the welled surface structures are calculated by the method described in Section I.A of Ref [11]. Briefly the scattered 
sound field is decomposed into plane wave scattering components, whose amplitudes are determined by the boundary condition at the welled surface. The method assumes a periodic boundary structure, and is therefore most accurate when there are large numbers of QRD periods. In our case there is only 1 QRD period, and the absorption coefficient calculated by this method will contain errors. However, the calculated absorption coefficients are only used as indications of the likely absorption ability of the barrier models in this study. The absorption coefficients are not used in the eventually BEM calculation of the barrier insertion loss, which uses directly the surface impedance boundary conditions. The experimental results described later in Section 7 show that the accuracy of the barrier insertion loss calculation is good enough for the current study.

\section{Numerical Model Results}

The absorption coefficients of the fibrous material and the different QRD constructions that are used in the barrier profiles were calculated using the above method. The surface is assumed to locally reacting. The absorption coefficients are averaged over full range of incident angle $\left(0<\theta_{e}<\pi / 2\right)$. The characteristics of the top surfaces used in this investigation are shown in Table 1. The insertion losses of the T- shape noise barriers with different top covers were then predicted using the 2D-boundary element method. The designs used in the simulations are also shown in Table 1.

The overall height of barriers is fixed at $3 \mathrm{~m}$, and the stem and cap thickness are respectively 0.1 and $0.3 \mathrm{~m}$ in all models. The barriers are located on rigid ground (the surface admittance is zero), and apart from the top surface all other surfaces of the barriers are rigid. The identification of each barrier indicates the property of its top surface. The configurations of all diffuser barriers can be found in Table 1. Two more barriers, a rigid $\mathrm{T}$ shape (all surfaces are rigid) and a reference absorptive $\mathrm{T}$ shape barrier, which were used in a previous paper [5] are also used here as references. The diffuser's overall span, which is $0.84 \mathrm{~m}$, is the same for all barrier models. A $0.08 \mathrm{~m}$ rigid frame is placed at both sides of the diffusers so that the overall span of the top surface is unity for 
all tested models in this investigation. The dimension of one of the tested diffuser designs, which is labelled model " $G$ " here, is shown in Figure 1.

Details of the perforated sheet, which is used in two different barriers (barrier model "GPT" and "GPI") is shown in Figure 2. Moreover the dimensions of the top surfaces of barriers model "GPT" (barrier model "G" covered with a layer of perforated sheet) and "GPI" ( barrier model "G" having a layer of perforated sheet inside its wells) are also shown in Figure 2.

The lower and upper frequency limit of the QRD structure can be estimated by the usual formulae [17]:

$\lambda_{\text {max }}=\frac{2 N d_{\text {max }}}{n_{\text {max }}} ; \lambda_{\text {min }} \approx 2 w$

where $d_{\max }$ is the maximum depth and $n_{\max }$ is the maximum number from the sequence $\left(n^{2} \bmod N\right)$. In this case the frequency bandwidth for the QRD used on the top surface of barrier model "G" should be from $400 \mathrm{~Hz}$ to $1.4 \mathrm{kHz}$. In practice the frequency bandwidth is found to be somewhat broader than this.

In all calculation in this paper the fin thickness is assumed to be negligible, but the viscous and thermal effect inside a well is taken into account.

\subsection{Top surface's absorbent coefficient}

Figure 3 to Figure 6 show the absorption ability of a few different diffusers that are used in the barrier models. The absorption coefficient of two different QR diffusers with the same frequency design but different well width is shown in Figure 3. As one can clearly see, reducing the well width increases the absorption coefficient of the diffuser because of increases in the viscous and thermal losses in the narrower wells. The peaks appear at similar frequencies but the use of narrower wells gives higher absorption at the upper end of the frequency range, as can be seen in the absorption coefficient frequency spectra. Figure 4 compares the absorption ability of two QR diffusers with different prime 
numbers but the same overall length. The QR diffuser with a smaller prime number and therefore fewer wells and wider well width has lower absorption coefficient. Primitive root diffuser is shown to have lower absorption than QR diffuser in Figure 5, mostly because of the smaller differences between adjacent wells and therefore smaller well coupling. According to Figure 6, the increase in the number of resonant frequencies in the diffuser with random sequence wells gives rise to more peaks in the absorption coefficient spectrum compare to a normal QR sequence. A common fact in all different tested diffusers is that the absorption ability is strongly frequency dependent because of the resonant behaviour.

Treating the surface of the QR diffuser, which is tuned to $400 \mathrm{~Hz}$ and has a well width of $12 \mathrm{~cm}$, with different surface covering materials and methods could considerably change the absorption coefficient of the device according to Figure 7 to Figure 9. Filling the wells by fibreglass (model "GF") and covering the surface by perforated sheet (model "GFP") in Figure 7 reduces the frequency dependency of the absorption coefficient of the diffuser while considerably increases in absorption in a wide frequency range. This in effect changes the QRD structure into a porous absorptive surface.

As expected, it is possible to increase the absorption coefficient of the diffuser dramatically by covering the top surface with a wire mesh $(\mathrm{R}=550$ Rayls (MKS)), as it is shown in Figure 8. However, in this case the frequency dependency due to well resonances can still be seen. Among the different wire meshes used, reducing the resisitivity of the wire mesh reduces the absorption ability of the covered diffuser except at the first resonant frequency. It is also worth noting that reducing the resisitivity of wire mesh increases the absorption coefficient fluctuation with frequency.

Using perorated sheets both at the top (model "GPT") and inside the wells (model "GPI"), as seen in Figure 9, shifts the region of high absorption of the diffuser towards lower frequencies and extends it to a wider frequency range. Although the absorption coefficient in the diffuser with perforated sheets inside the wells is lower than that of the 
diffuser with perforated sheet on the top at lower frequencies, the perforated sheets inside the wells create more resonances and produce more absorption peaks.

It should be noted the positions of the perforated plates in the wells are important for the resonant frequencies according to equations 8 and 9 . The first resonant frequency can be changed by adjusting the position of the perforated sheets in the wells. Such optimisation is however beyond the scope of this study.

\subsection{QRD edged barriers treated with fibreglass, perforated sheet and wire mesh}

As expected, Figure 6 shows that using fibreglass inside the wells increases the absorption coefficient of QR diffuser. However, our interest is to see how this increase in absorption affects the performance of a QRD barrier. Figure 10 shows to what extent the absorption ability of a diffuser changes the performance of the QRD barrier. Increasing the top surface absorption coefficient of a diffuser barrier by fibreglass in model "GF" actually reduces the insertion loss over the entire frequency bandwidth of the QRD, although slight improvement is shown outside the frequency limits when the QRD is inactive. In fact the effect of resonant wells almost vanishes because of the large increase in absorption, so much so that the new surface simply acts like a normal absorbent surface. In Figure 11 when the top surface of the QR diffuser utilised in model "G" is covered with a perforated sheet to become model "GPT", the increase in absorptive resistance is smaller and the resonant wells are still effective though not as strong as in the original model " $G$ ", as is evident in the insertion loss frequency spectrum. The effective frequency band also shifts slightly to lower frequencies.

In contrast, covering the top surface of the $\mathrm{QRD}$ by a highly resistive wire mesh $(\mathrm{R}=550$ Rayls (MKS)) in model "GWH" reduces the effectiveness of the QRD on the top surface of T- shape barrier, as can be seen in Figure 12. In fact the behaviour becomes similar to that of filling the wells with fibreglass, since both produce high absorptive resistance in 
the wells that effectively kills off the resonances (Figures 7 and 8). Thus the advantage of the diffuser is lost, and the performance tends towards a purely absorptive barrier.

\subsection{IL differences created by absorptive treatments on model " $G$ "}

In order to give a clear demonstration of the results for different barrier designs, the insertion loss of the $\mathrm{T}$ profile barriers with various well covers are compared with that of the QR diffuser barrier model " $G$ " that has no covers. The overall configurations of all barriers are the same except that some diffusers have extra treatments such as wire mesh or perforated sheets either at the top or inside the wells to increase the absorption. It has been shown in [5] that the barrier model " $G$ ", which is a QRD edged barrier tuned to 400 $\mathrm{Hz}$ with a $12 \mathrm{~cm}$ well width, has higher performance than rigid and purely absorptive $\mathrm{T}$ shape barrier from about $250 \mathrm{~Hz}$ in a wide frequency range. However outside the frequency limit of the diffuser the amount of improvement was very small to negative. The effect of reducing well width was also investigated in reference [5] by the authors. It was found that reducing the well width did not improve the overall performance of the profile barriers. The purpose here is to see if the performance of the QRD barrier model can be improved by increasing its absorption.

Figure 13 shows that a primitive root sequence diffuser (PRD) barrier (model "GPR") has higher performance than a QRD edge barrier (model "G") at lower frequencies even inside the frequency bandwidth of the QRD, although the reverse is seen at frequencies between $800 \mathrm{~Hz}$ till $2 \mathrm{kHz}$. The diffuser in the random sequence model "GR", which has more resonant frequencies provided by the additional variations of well depths, shows some higher efficiency over both the QRD and PRD barrier models at mid frequencies, although its efficiency is lower at the low frequencies $(200-630 \mathrm{~Hz})$ and becomes similar to that of the PRD model at frequencies higher than about $2 \mathrm{kHz}$. This shows that the efficiency of a diffuser barrier is highly dependent on the resonant behaviours of the diffuser top. More and stronger resonances tend to give better performances.

Figure 14 shows the effect of putting wire meshes of various resistivity on top of the diffuser on the efficiency of the QRD edge barriers. The results are shown relative to an 
equivalent purely absorbent $\mathrm{T}$ shape barrier. Increasing the resistivity of the tested wire meshes slightly improves the performance of the barrier just outside the frequency bandwidth of the diffuser, but the efficiency of the barriers decreases significantly within the design frequency range of the diffuser when the resistance of the wire mesh is increased. Similarly, Figure 15 shows that the effect of the diffuser almost disappeared when the wells are filled with highly absorptive fibreglass ( barrier model "GF"). Covering the top surface further with a perforated sheet (barrier model "GFP") gives similar results.

The effect of putting a perforated sheet either on the top or inside the wells is shown in Figure 16. It is seen that the peaks slightly shift toward lower frequencies when the perforated sheet is implemented at the top surface of the QRD (barrier model "GPT"), but the overall performance remains similar. This is possibly due to the fact that the dissipative resistance of the perforated sheet, which has $2.5 \mathrm{~mm}$ diameter holes and 19.6 perforation ratio, is not large at frequencies below the maximum frequency shown in the figure. The effect of the perforated sheet is reactive more than resistive. Thus the well resonances are preserved amid a shift in the resonant frequencies.

\subsection{Broad band insertion loss}

The A- weighted road traffic noise spectrum is used to weight the results for insertion loss at one-third octave band centre frequencies over the range 50 to $4000 \mathrm{~Hz}$ [18]. It is done to average out the strong interference effects observed at mono-frequencies, and to allow trends to be identified more easily. The broadband insertion loss averaged over nine receiver positions $-20,50$ and $100 \mathrm{~m}$ from the centre line of the barrier on the ground and at 1.5 and $3 \mathrm{~m}$ above the rigid ground and A-weighted with the traffic noise spectrum are shown in Table 2. The differences in the mean insertion loss relative to the equivalent rigid T-shape barrier are also shown (labeled $\Delta \mathrm{IL}$ ).

All the top surface treatments are shown to improve the A-weighted insertion loss of the T-shape barrier apart from model "I", which is the QRD barrier with extremely narrow wells. Although, according to Figure 3, reducing the well width of the applied QRD 
increases the absorption coefficient of the device, it is shown here that it has a negative effect on the A-weighted insertion loss of T-shape QRD barriers. In this case, reducing the well width in a QRD from $12 \mathrm{~cm}$ in model " $\mathrm{G}$ " to $6 \mathrm{~cm}$ in model " $\mathrm{H}$ ", and to $2 \mathrm{~cm}$ in model "I" decreases the A-weighted insertion loss of the barriers from $19 \mathrm{~dB}$ (A) to 18.3 $\mathrm{dB}(\mathrm{A})$ and to $16.7 \mathrm{~dB}(\mathrm{~A})$ respectively.

The QRD edge barrier (model "G") improves the A-weighted insertion loss by $0.9 \mathrm{~dB}(\mathrm{~A})$ over that of an equivalent purely absorptive $\mathrm{T}$-shape barrier. Reducing the number of wells in resonance by using a QRD with less prime number, as in model "J", causes lower A- weighted IL - by $1.1 \mathrm{~dB}$ (A) compare to model "G" which has a higher number of wells. The random well sequence in model "GR" improves the performance of rigid Tshape barrier by only $0.4 \mathrm{~dB}(\mathrm{~A})$ so is not effective. The primitive root sequence in model "GPR" does however improve the A - weighted insertion loss of the model "G" slightly by $0.3 \mathrm{~dB}(\mathrm{~A})$.

The best treatment on the QRD is seen to be putting a perforated sheets inside the wells, which improves the A-weighted insertion loss of barrier model " $G$ " by $0.6 \mathrm{~dB}(\mathrm{~A})$. In contrast filling the wells by fiberglass is the worst treatment on QRD barriers. In this case the barrier model "GF", with a mean A-weighted insertion loss of $18 \mathrm{~dB}(\mathrm{~A})$, has $1 \mathrm{~dB}$ (A) less performance than model "G". These best and worst results show the importance of resonances rather than absorption in a diffuser barrier. Using a perforated cover shifts the resonant frequencies by adding mass to the system, creating Helmholtz resonator effects with the air cavities of the wells behind it. It is worth adding that in this research the perforated sheet and its placement within the wells have not been specially optimized. It means that it may be possible to improve the design by further optimization to achieve even better performance of a diffuser covered barrier. This will be an interesting further work.

\subsection{Sound field at the shadow zone}


Details of the far and near field effect of a selected high performance diffuser barrier, model "GPI", are compared with that of the plain QRD barrier model "G". The broadband differences of the A-weighted insertion loss of barrier model "GPI" relative to the plain QRD barrier model "G" at 400 receiver positions is plotted in Figure 17. It is worth adding that the authors [5] have shown that model " $G$ " has better performance in a very wide area both in the near and far field and close and above the ground compare to equivalent rigid and purely absorptive T-shape barriers. According to [5] the amount of far field improvements made by barrier model " $G$ " compare to an equivalent purely absorptive T shape barrier at heights of 6 to $8 \mathrm{~m}$ was between 2.5 to $3.5 \mathrm{~dB}(\mathrm{~A})$, while the same amount of improvement was shown at zones close to the ground in both near and far field.

In Figure 17, the improvement by introducing perforated sheet inside the wells of the QRD barrier is shown. Although in the far field the QRD with no perforated sheet (model "G") has better performance, model "GPI" improves the performance of model "G" in the near filed especially at higher height, which is the weak area of the plain QRD due to probably its upward sound diffraction. It seems that the perforated sheet reduces the upward diffraction of diffuser by introducing higher absorption coefficient especially at frequencies below the design frequency bandwidth.

\section{Scale Modelling Results}

The results obtained in the previous sections were obtained only by numerical calculations. Although the numerical method has already been found in previous papers to have very good agreement when applied to rigid, absorptive, and reactive profiled barriers $[2,16,19]$, and to Schroeder diffusers at normal incidence [17], its accuracy when dealing with a diffuser on a profile barrier needs to be confirmed experimentally. It was done here using physical scale measurements.

The scale used in the experiments was 1:4. Thus, the full-scale frequency range $50 \mathrm{~Hz}-4$ $\mathrm{kHz}$ relates to $200 \mathrm{~Hz}-16 \mathrm{kHz}$ in the scale model ( the typical geometry used is shown 
in Figure 18). All the surfaces were constructed from 18-mm chipboard with a surface density equal to $11.8 \mathrm{kgm}^{-2}$. In the QRD barriers the QRD top is a sandwich construction of 18-mm chipboard and $3.2 \mathrm{~mm}$ hardwood. All the surfaces including the inside walls of the wells were well varnished to reduce surface absorption. The flow resistivity of the resistive layer used in the wire meshed QRD barrier was 315 ralys (MKS). A maximum length sequence [20,21] system was used to measure the time domain sound pressure impulse responses at the receiver position.

In Figure 19, the results of the scale model experiments for four different barriers are shown. Good agreement between measurement and the BEM prediction can be seen over a wide frequency range for all the different barriers that were tested. In most cases the measurement results were slightly higher than predicted results. Although in the single rigid and $\mathrm{T}$ shape barrier cases the result of the prediction were rather smooth, the result for the QRD barrier showed some fluctuations mainly at higher frequencies. The amount of fluctuations in the QRD barrier covered with wire mesh is slightly less than that of the plain QRD barrier. Importantly, the experiment shows no significant improvement by utilizing wire mesh on the top of the QRD barrier, as predicted by the BEM.

\section{Discussion and conclusion}

The performance of welled diffuser edged T-shape noise barriers with absorptive treatments has been investigated using a two-dimensional boundary element method. Absorption coefficient was changed by treating the QRD with fiberglass and perforated sheets. Broadband insertion loss, weighted with a standard traffic noise spectrum, has been predicted over a range of receiver positions using a spectrum of 1/15-octave band from $50 \mathrm{~Hz}$ to $4000 \mathrm{~Hz}$. The performance of a few different top surfaces - rigid; absorptive; and different welled diffusers with and without treatments, on the T- shape barrier has been evaluated. The welled diffusers investigated include a QR diffuser, a PR diffuser and a Random sequence diffuser. In some models the surface and wells of the QR diffuser were treated with fiberglass, wire mesh and/or perforated sheets. 
The performances of the barriers have also been compared with their equivalent absorbent and rigid barriers, as well as compared with the selected plain QRD barrier without cover. The results can be summarised as follows:

1. Reducing the well width increases the absorption coefficient of diffusers because of viscous and thermal effects in the narrower wells. It is shown that changing the diffuser sequence from $\mathrm{QR}$ to $\mathrm{PR}$ decreases the absorption coefficient of the diffuser slightly, possibly as a result of the reduced number of non-zero well depths (5 in PR compared with 6 in QR in the case studied here). Increasing the number of resonant frequencies by changing the $\mathrm{QR}$ sequence to Random sequence raises the number of peaks in the absorption spectrum.

2. Implementing wire mesh, fiberglass, and/or perforated sheet could increase the absorption coefficient of the QRD substantially. A wire mesh or fiberglass with high resistivity reduces the effect of wells in resonance, therefore the spectrum of absorption coefficient shows less fluctuations. In contrast perforated sheet could increase the absorption coefficient of the QRD as well as lower the resonant frequencies of the diffuser.

3. Putting high resistivity fiberglass or wire meshes in the QRD top of a diffuser barrier significantly reduces the performance of the barrier within the frequency bandwidth of the diffuser despite the large increase in absorption. This is because of the reduction or even removal of the effect of well resonances, which is the main factor contributing to the good performance of a QRD barrier.

4. Putting perforated sheets inside the wells of a QRD barrier (model "GPI") improves the broadband, traffic noise weighted performance of the QRD barrier by $0.6 \mathrm{~dB}(\mathrm{~A})$. This is because of the lowering of the well resonant frequencies of the diffuser. 
5. The use of perforated sheets inside the QRD wells also improves the relatively weak performance of a QRD edge barrier at receivers close to and above the top surface of the barrier (at receivers above $4 \mathrm{~m}$ close to the barrier in our case) by up to $1.6 \mathrm{~dB}(\mathrm{~A})$. However it also reduces the strength of the QRD edge barrier at receivers close to ground slightly by about $-0.4 \mathrm{~dB}(\mathrm{~A})$.

6. Good accuracy of the 2D prediction methods over a wide range of frequency was confirmed by 1:4 scale model measurements. Furthermore the experiment also confirms the finding from numerical simulations that no significant improvements in barrier insertion loss can be achieved by employing highly resistive materials on the top surface or inside the QRD to increase the absorption of a QRD barrier.

The investigation in this paper on the effect of increasing the absorption properties of diffusers implemented on T-shape barriers has clearly shown that, although it is good for normal barriers to increase the absorption, any absorption gain as a result of decreasing the well resonance effect in a QRD is harmful to the efficiency of QRD barriers. 


\section{$\underline{\text { References }}$}

1. T. Okubo, K. Fujiwara. "Efficiency of a noise barrier with an acoustically soft cylinder edge for practical use”, J. Acoust. Soc. Am . 105 (6), pp.3226-3335, June 1999.

2. D.C. Hothersall, D.H. Crombie, and S.N. Chandler-Wilde, "The performance of Tshape profile and associated noise barriers", Applied Acoustics 32(4), pp.269-281, 1991.

3. D. C. Hothersall, S. N. Chandler-Wilde and M. N. Hajmirzae, "Efficiency of single noise barriers", Journal of Sound Vibration, 146(2), pp.303-322, 1991.

4. K. Fujiwara." Sound shielding efficiency of a barrier with soft surface", Proc.Internoise, 90, pp 343 -346, 1990.

5. M. R. Monazzam and Y. W. Lam, "Performance of profile single noise barriers covered with quadratic residue diffusers”, Applied Acoustics 66, pp.709-730, 2005.

6. D. E. Commins, N. Auletta, B. Suner, "Diffusion and absorption of quadratic residue diffusers", Proc. I. O. A, 10 (2), pp 223-232, 1988.

7. K. Fujiwara., T. Miyajima "Absorption characteristics of a practically constructed Schroeder diffuser of quadratic- residue Type", Applied Acoustics 35, pp.149-152, 1992.

8. H.Kuttruff, "Sound absorption by pseudostochastic diffusers (Schroeder Diffuser)", Applied Acoustics 42, pp.215-231, 1993.

9. K. Fujiwara." A study on the sound absorption of a quadratic- residue type diffuser", Acoustica, 81, pp 370 -378, 1995.

10. F.P.Mechel." The wide-angle diffuser- a wide- angle absorber?” Acoustica, 81, pp $379-401,1995$.

11. T. Wu, T. J. Cox, and Y. W. Lam, "From a profiled diffuser to an optimised absorber", J. Acoust. Soc. Am .108 (2), pp.643-650, 2000

12. T. Wu, T. J. Cox, and Y. W. Lam, "A profiled structure with improved low frequency absorption”, J. Acoust. Soc. Am .. 119 (6), pp.3064-3070, 2001

13. M. R. Schroeder, "Diffuse sound reflection by maximum length sequence", J. Acoust. Soc. Am . 57 (1), pp.149-150, Jan 1975. 
14. M. R. Schroeder, "Binaural dissimilarity and optimum ceilings for concert halls: More lateral sound", J. Acoust. Soc. Am . 65 , pp.958-963, 1979.

15. M. E. Delany and E. N. Bazely, "Acoustical properties of fibrous absorbent material", Applied Acoustics 3(2), pp.105-116, 1970.

16. K. Fujiwara, D. C. Hothersall and C. H. Kim, "Noise barriers with reactive surfaces", Applied Acoustics, 53 (4), pp. 225-272, 1998.

17. T. J. Cox and Y. W. Lam, "Prediction and evaluation of the scattering from quadratic residue diffusers", J. Acoust. Soc. Am. 95 (1), pp.297-305, 1994.

18. BS EN 1793-3:1998, Road traffic noise reducing devices- Test method for determining the acoustic performance, Part 3. Normalized traffic noise spectrum.

19. D.H. Crombie, D.C. Hothersall, and S.N. Chandler-Wild, "Multiple-edge noise barrier", Applied Acoustics 44(4), pp.353-367, 1995.

20. Borish J and Angell J.B, “ An efficient algorithm for measuring the impulse response using pseudo random noise", Journal of Audio Engineering Society 31(7), 477-488, 1982.

21. Rife D.D and Vanderkooy J, "Transfer-function measurement with maximum-length sequences”, Journal of Audio Engineering Society 37, 419-444, 1989. 
Table 1 Design model names and corresponding configurations.

\begin{tabular}{|c|c|c|c|c|c|c|}
\hline Models & $\mathrm{N}$ & $\begin{array}{c}\text { Design } \\
\text { Frequency } \\
f_{r}(\mathrm{kHz})\end{array}$ & $\begin{array}{c}\text { Well } \\
\text { width } w \\
\text { (cm) }\end{array}$ & $\begin{array}{l}\text { Diffuser } \\
\text { type }\end{array}$ & Sequence* & Description \\
\hline$G$ & 7 & 0.4 & 12 & QR & 0142241 & No treatment \\
\hline $\mathrm{H}$ & 7 & 0.4 & 6 & QR & $\begin{array}{lllllll}0 & 1 & 4 & 2 & 2 & 4 & 1 \\
0 & 1 & 4 & 2 & 2 & 4 & 1\end{array}$ & No treatment \\
\hline $\mathrm{I}$ & 7 & 0.4 & 2 & QR & $\begin{array}{c}5 \text { periods of } \\
(0142241) \\
\text { and } \\
1422410 \\
\end{array}$ & No treatment \\
\hline $\mathrm{J}$ & 5 & 0.56 & 16.8 & QR & 01441 & No treatment \\
\hline GF & 7 & 0.4 & 12 & QR & 0142241 & $\begin{array}{c}\text { "G" with fiberglass in } \\
\text { wells }\end{array}$ \\
\hline GFP & 7 & 0.4 & 12 & QR & 0142241 & $\begin{array}{l}\text { "G" with fiberglass and } \\
\text { Perforated sheet** (top) }\end{array}$ \\
\hline GWH & 7 & 0.4 & 12 & QR & 0142241 & $\begin{array}{c}\text { "G" with wire mesh(R= } \\
550 \text { Rayls(MKS)) }\end{array}$ \\
\hline GWM & 7 & 0.4 & 12 & QR & 0142241 & $\begin{array}{l}\text { "G” with wire mesh } \\
(\mathrm{R}=55 \text { Rayls }(\mathrm{MKS}))\end{array}$ \\
\hline GWL & 7 & 0.4 & 12 & $\overline{Q R}$ & 0142241 & $\begin{array}{l}\text { "G” with wire mesh } \\
(\mathrm{R}=5.7 \text { Rayls (MKS)) }\end{array}$ \\
\hline GPT & 7 & 0.4 & 12 & QR & 0142241 & $\begin{array}{c}\text { "G" with perforated } \\
\text { sheet**(top) }\end{array}$ \\
\hline GPI & 7 & 0.4 & 12 & QR & 0142241 & $\begin{array}{l}\text { "G” with perforated } \\
\text { sheet(inside)*** }\end{array}$ \\
\hline GR & 7 & 1 & 12 & $\begin{array}{l}\text { Random } \\
\text { sequence }\end{array}$ & 01357910 & No treatment \\
\hline GPR & 6 & 0.4 & 14 & $\begin{array}{l}\text { Primitive } \\
\text { root } \\
\text { sequence } \\
\end{array}$ & 215340 & No treatment \\
\hline
\end{tabular}

* $d_{\text {max }}$ is $0.2445 \mathrm{~m}$ in entire models apart from model “GPR” which it is $0.3 \mathrm{~m}$.

** $5 \mathrm{~mm}$ thick, holes are of $2.5 \mathrm{~mm}$ diameter and spaced at $5 \mathrm{~mm}$ centre to centre. Percentage of open over full area is $19.6 \%$.

*** Perforated sheets are located inside the wells randomly at the following heights above the bottom of each well according to the mentioned well sequence $(0,2,9,10,5,21,6.1 \mathrm{~cm})$. 
Table 2 Broadband insertion losses averaged over nine receivers for various forms of barriers. The values are A-weighted with a standard traffic noise spectrum.

\begin{tabular}{|l|c|c|}
\hline & \multicolumn{2}{|c|}{ Broad band IL over 9 receivers (dB(A)) } \\
\hline Barrier type & Mean & $\Delta \mathrm{L}$ \\
\hline T shape & 16.9 & 1.2 \\
\hline A T shape & 18.1 & 2.1 \\
\hline G & 19 & 1.4 \\
\hline H & 18.3 & -0.2 \\
\hline I & 16.7 & 1 \\
\hline J & 17.9 & 1.1 \\
\hline GF & 18 & 1.3 \\
\hline GFP & 18.2 & 2.2 \\
\hline GWH & 19.1 & 2.2 \\
\hline GWM & 19.1 & 2.1 \\
\hline GWL & 19 & 2.3 \\
\hline GPT & 19.2 & 2.7 \\
\hline GPI & 19.6 & 0.4 \\
\hline GR & 17.3 & 2.4 \\
\hline GPR & 19.3 & 0.1 .5 \\
\hline
\end{tabular}

Receiver positions are: 1 at $(20.0) ; 2$ at $(50,0) ; 3$ at $(100,0) ; 4$ at $(20,1.5) ; 5$ at $(50,1.5) ; 6$ at $(100,1.5) ; 7$ at $(20,3) ; 8$ at $(50,3) ; 9$ at $(100,3)$ and $\Delta \mathrm{IL}$ is the difference between the mean insertion loss of a barrier design and the mean insertion loss of a reference rigid T-Shape barrier. Source position is at $(-5,0)$. For the absorptive case (type with prefix "A") the upper surface of the cap is covered with an absorbent material with $\sigma=20000$ Rayls (MKS) and thickness of $0.2445 \mathrm{~m}$. QRD barrier types are as described in Table 1. 


\section{Figure Titles:}

Figure 1 Dimensions of the T- shape barrier having a QRD ( $N=7$ and $\left.f_{r}=0.4 \mathrm{kHz}\right)$ labelled model "G”.

Figure 2 Dimensions of top surface of barriers models "GPT" (barrier model "G” having a layer of perforated sheet on top) and "GPI" (barrier model "G" having a layer of perforated sheet inside each well) along with a cross section of the utilized perforated sheet.

Dimensions of barrier model " $G$ " is shown in Figure 1.

Figure 3 Comparison of the predicted absorption coefficients of a QR diffuser $(\mathrm{w}=12 \mathrm{~cm})$ used in the barrier model " $G$ " and a QR diffuser $(w=2 \mathrm{~cm})$ used in the barrier model "I".

Figure 4 Comparison of the predicted absorption coefficients of a QR diffuser $(\mathrm{N}=7)$ used in the barrier model "G” and a QR diffuser $(\mathrm{N}=5)$ used in the barrier model “J”.

Figure 5 Comparison of the predicted absorption coefficients of a QR diffuser used in the barrier model "G" and a PR diffuser used in the barrier model "GPR".

Figure 6 Comparison of the predicted absorption coefficients of a QR diffuser used in the barrier model "G” and a random sequence diffuser used in the barrier model "GR".

Figure 7 Absorption coefficients of the top surface of the model "G", model "GF" and model "GFP" barrier.

Figure 8 Comparison of the predicted absorption coefficients of a QRD covered with different wire meshes, as in the barrier models "GWH”, "GWM" and "GWL”, with wiremesh resistivity of 550 , 55 and 5.7 (MKS Ralys) respectively.

Figure 9 Comparison of the predicted absorption coefficients of a QR diffuser treated with perforated sheets, as in the barrier models “GPT” (top) and “GPI” (inside).

Figure 10 Comparison of the insertion loss of the QRD barrier model "G" and barrier model "GF” which has the QRD filled with fibreglass. The receiver point is at $(-50,0)$.

Figure 11 Comparison of the insertion loss of the QRD barrier model "G" and barrier model "GPT" which has the QRD top surface covered with a perforated sheet. The receiver point is at $(-50,0)$.

Figure 12 Comparison of the insertion loss of the QRD barrier model "G" and barrier model "GWH" which has the QRD top surface covered with a wire mesh ( $\mathrm{R}=550 \mathrm{MKS}$ Rayls). The receiver point is at $(-50,0)$.

Figure 13 Extra insertion loss provided by the plain QRD barrier model " $G$ ” relative to model “GPR” (primitive root) and "GR” (random sequence) barriers at the receiver point ($50,0)$.

Figure 14 Extra insertion loss provided by the plain QRD barrier model " $G$ " and the wire meshed QR diffuser models “GWH”, “GWM” and "GWL” (wire-mesh resistivity R= 550, 55 
and 5.7 (MKS Ralys) respectively) relative to an equivalent purely absorptive T- shape barrier (model “AT”) at the receiver point $(-50,0)$.

Figure 15 Extra insertion loss provided by the barrier models "G” (plain QRD), "GF” (filled with fibreglass) and "GFP" (filled with fibreglass and covered with a perforated plate) relative to an equivalent purely absorptive T- shape barrier (model "AT") at the receiver point $(-50,0)$.

Figure 16 Extra insertion loss provided by the barrier models "G” (plain QRD), “GPT” (covered with a perforated plate) and "GPI" (treated with perforated sheets at different depths inside the wells) relative to an equivalent purely absorptive T- shape barrier (model "AT") at the receiver point $(-50,0)$.

Figure 17 Contour plot of differences of broad band A- weighted insertion loss of barrier model “GPI” relative to the plain QRD barrier model "G” calculated over 400 receiver positions.

Figure 18 The geometry of the T shape and QRD barrier in the physical scale experiment. All dimensions are given at the 1:4 scale. "L" , " $\mathrm{H}$ " and "W" represent the length, height and width of the relevant parts of the models.

Figure 19 Measured and predicted spectra for four different barrier models. The overall dimensions of the barrier, and the source and receiver positions are the same in each case (see Figure 18). __ Experimental measurement; - - - boundary element model prediction. 


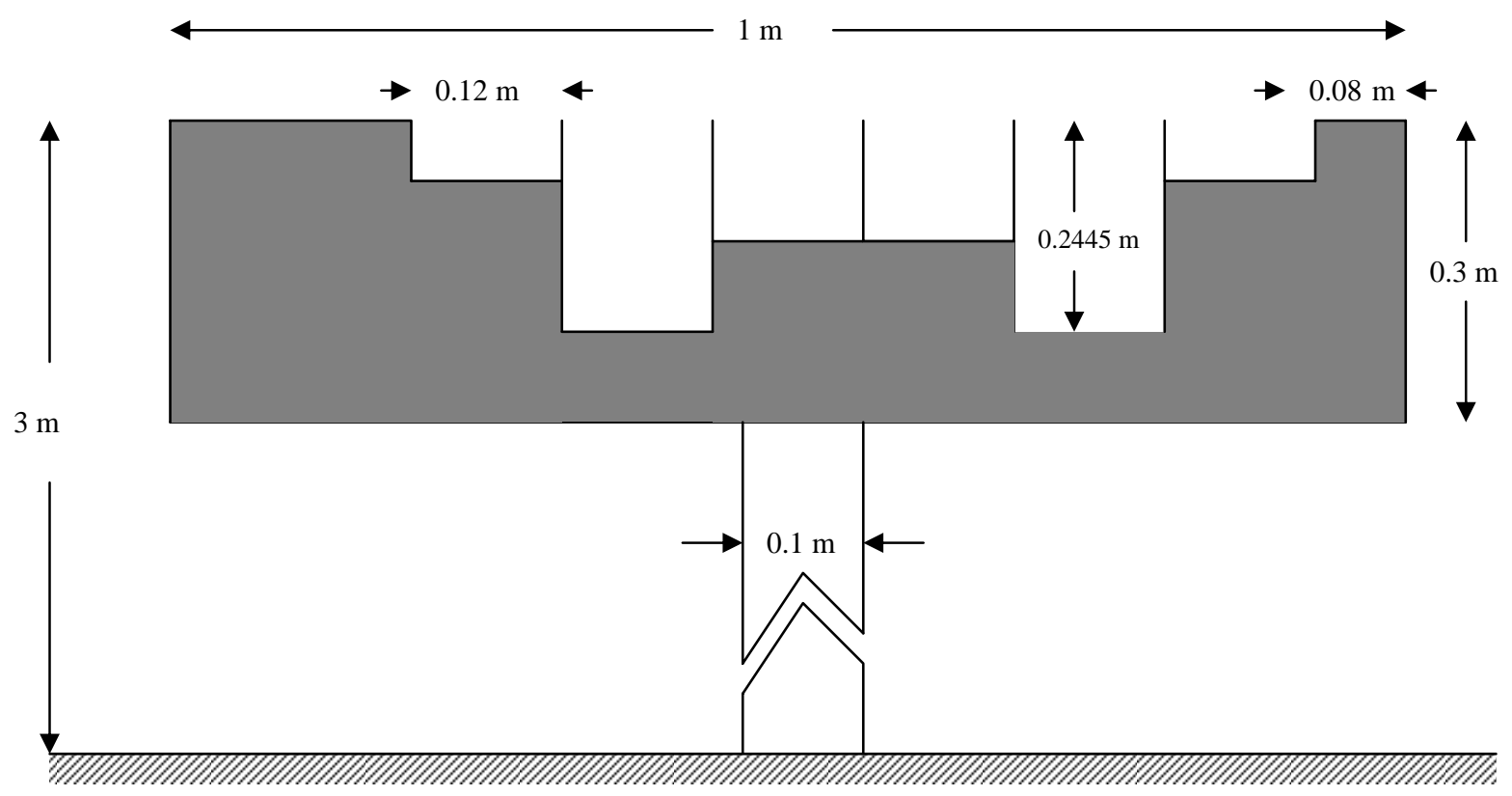




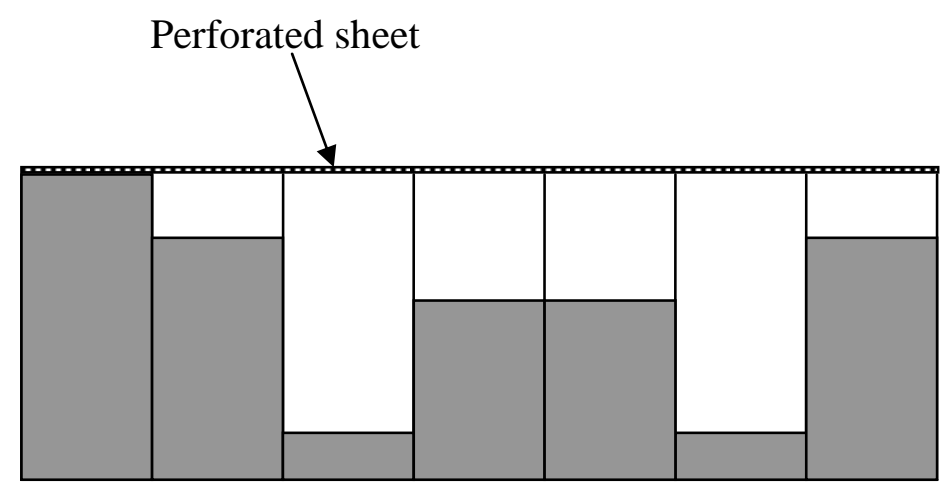

Top surface of barrier model "GPT"

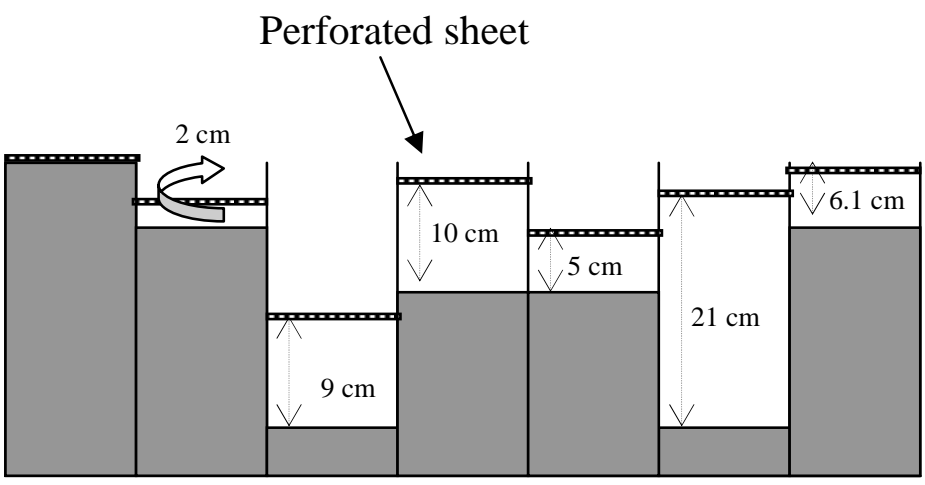

Top surface of barrier model "GPI”

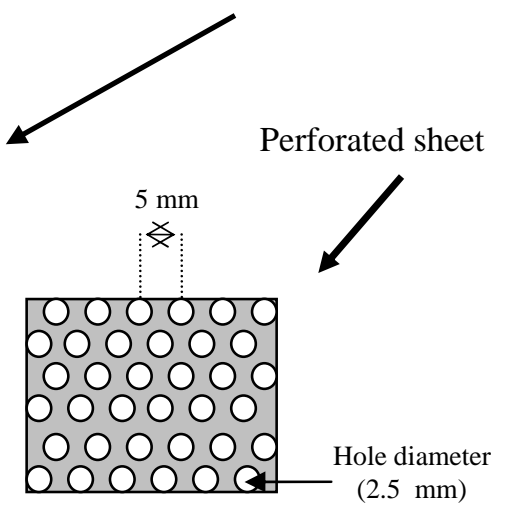




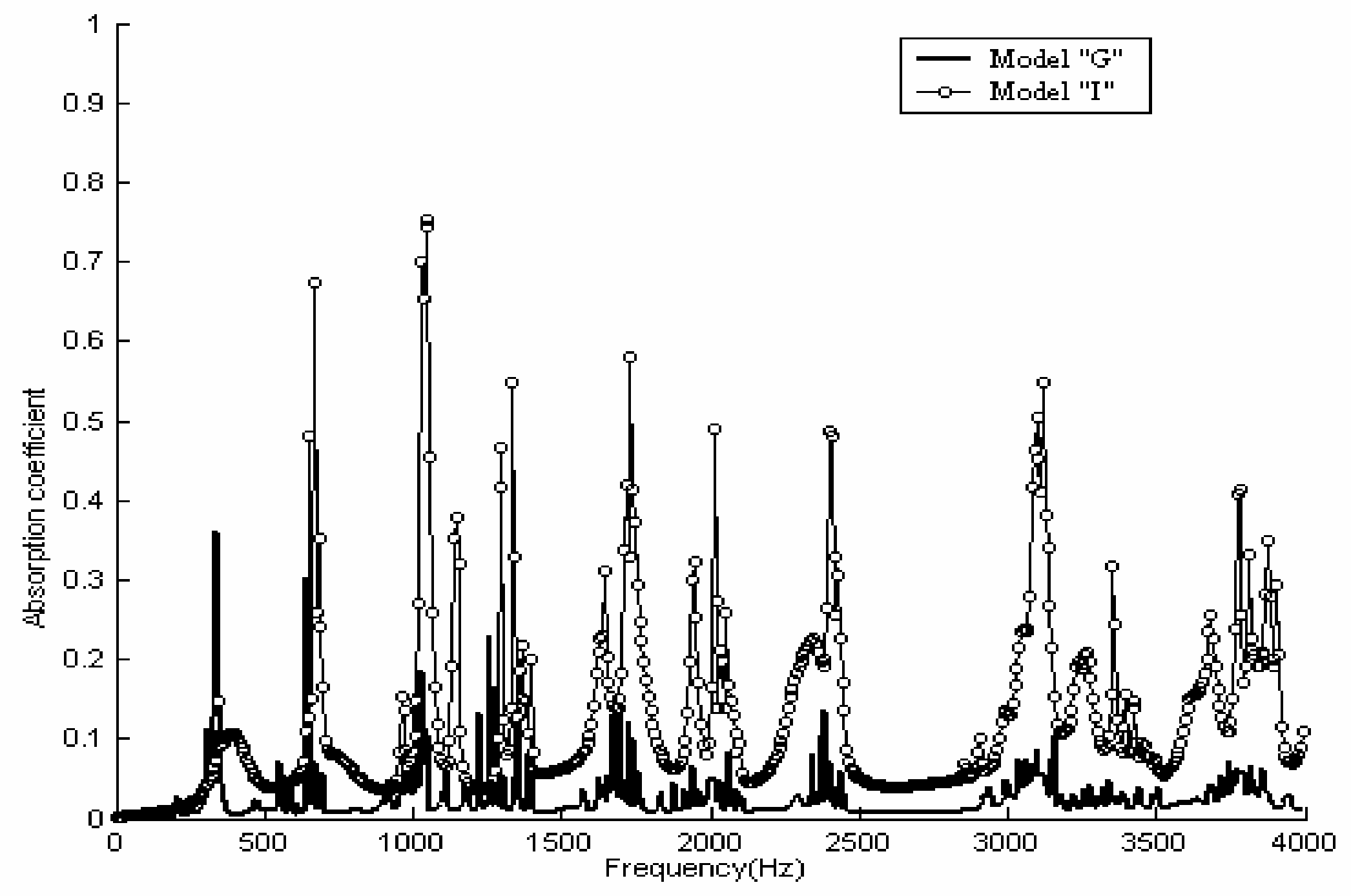




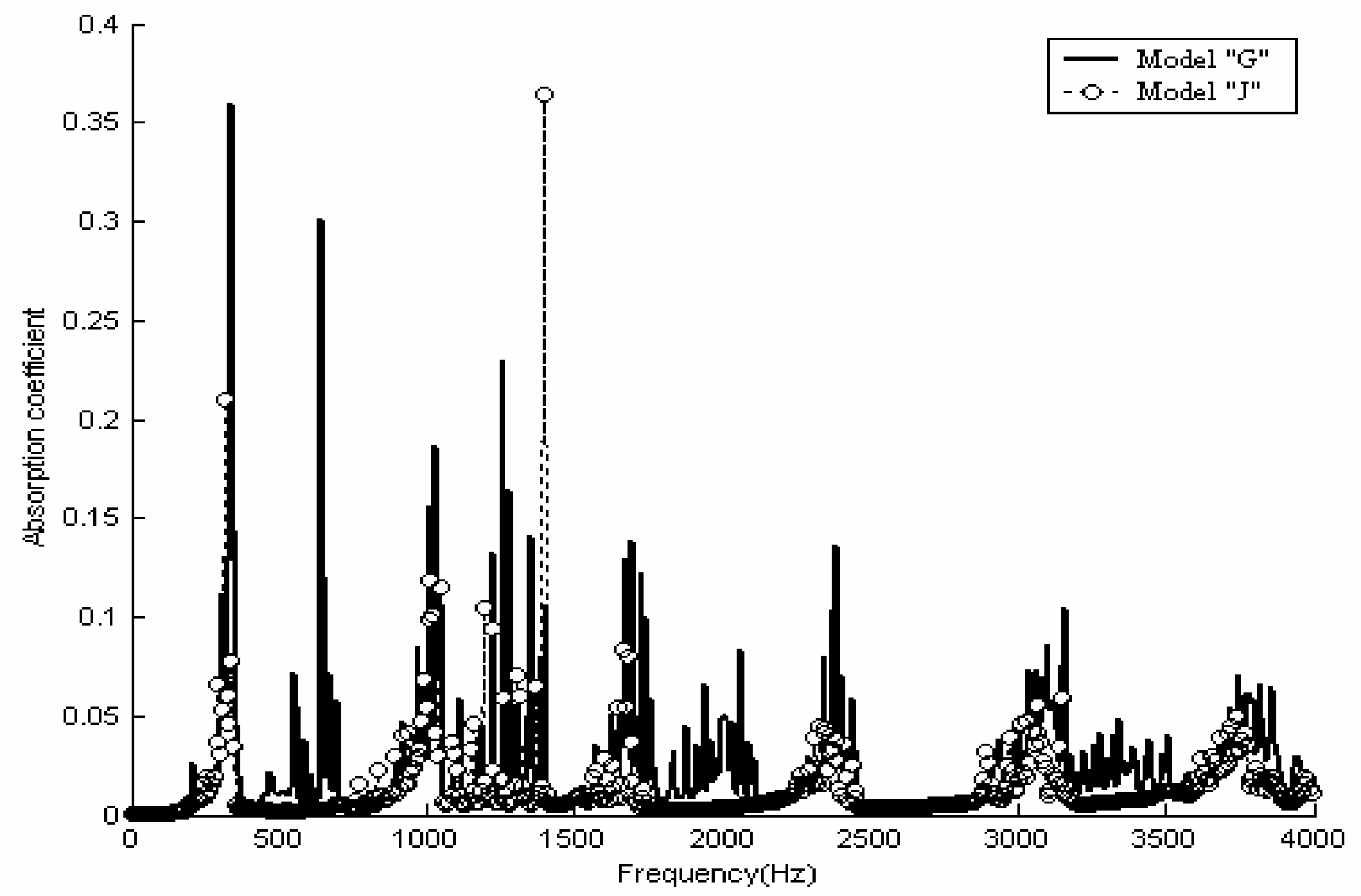




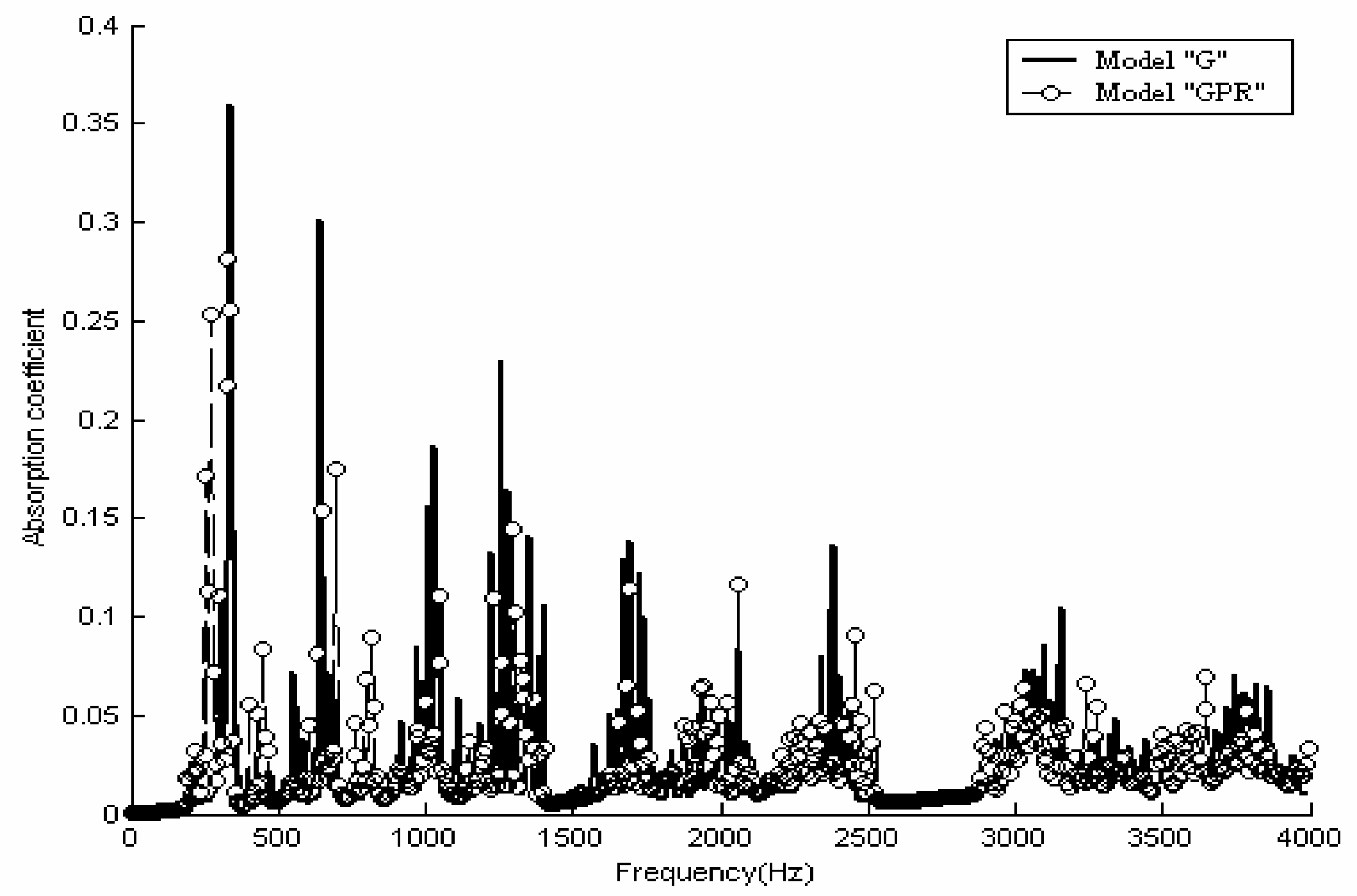




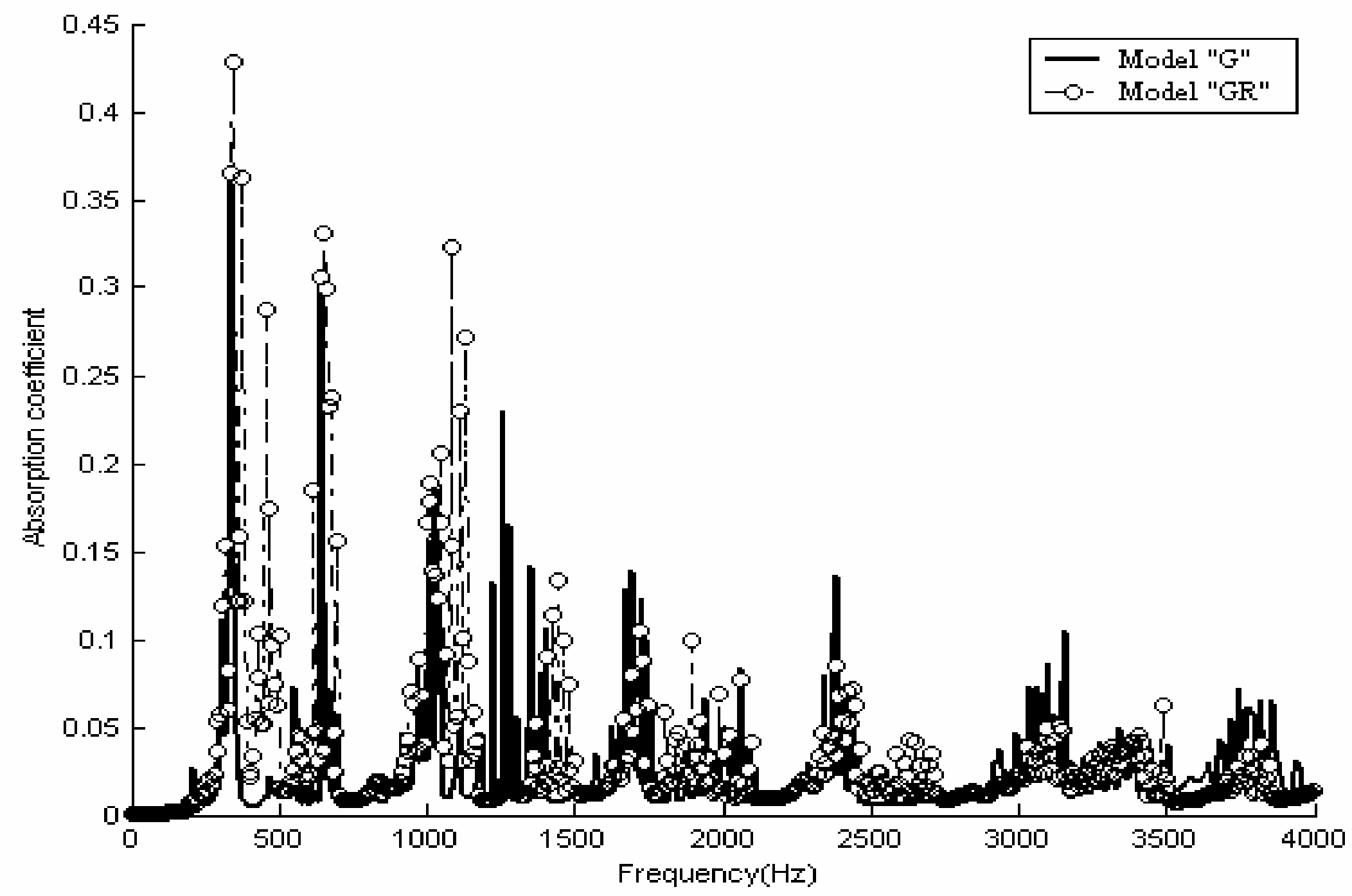




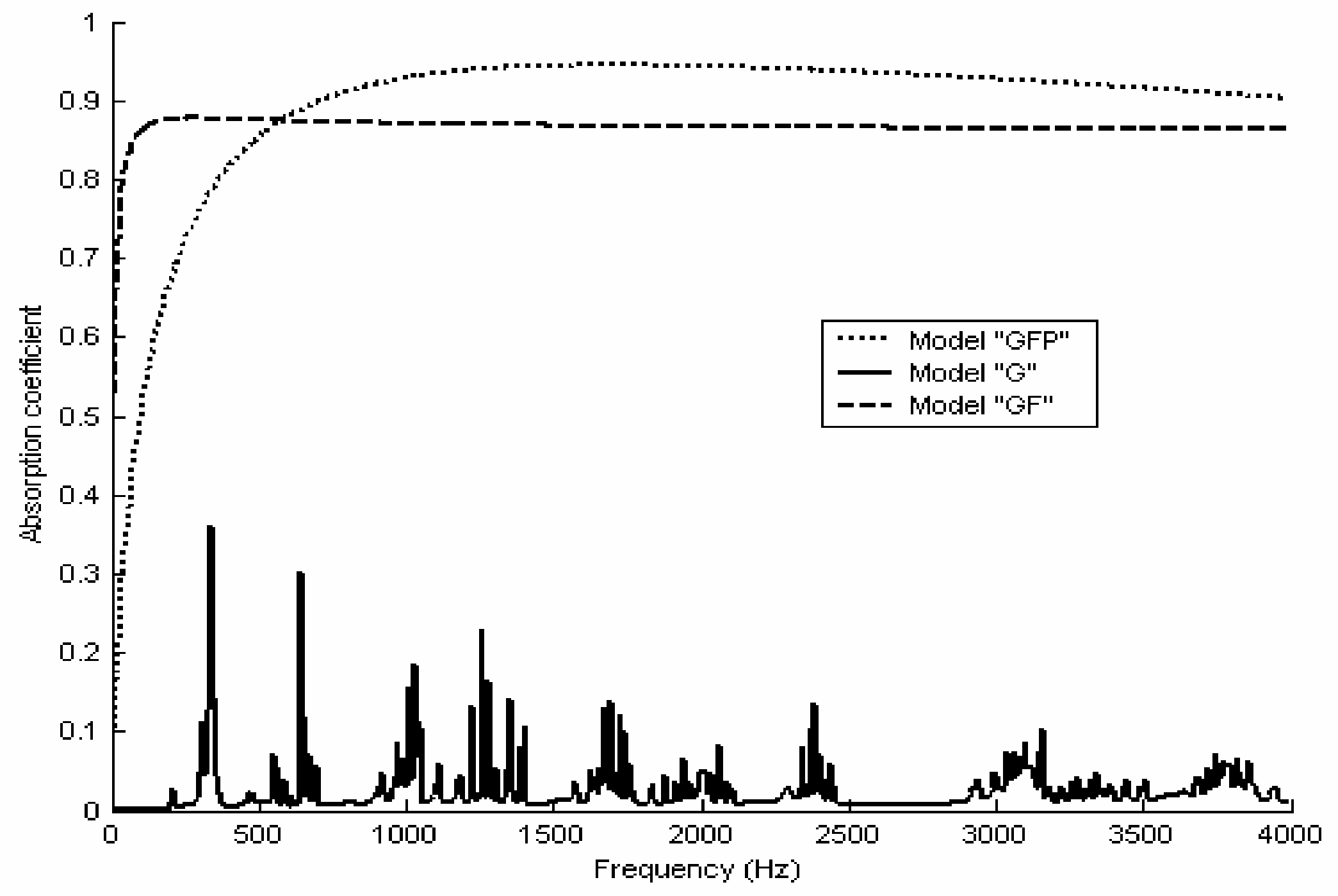




$$
\text { mer }
$$




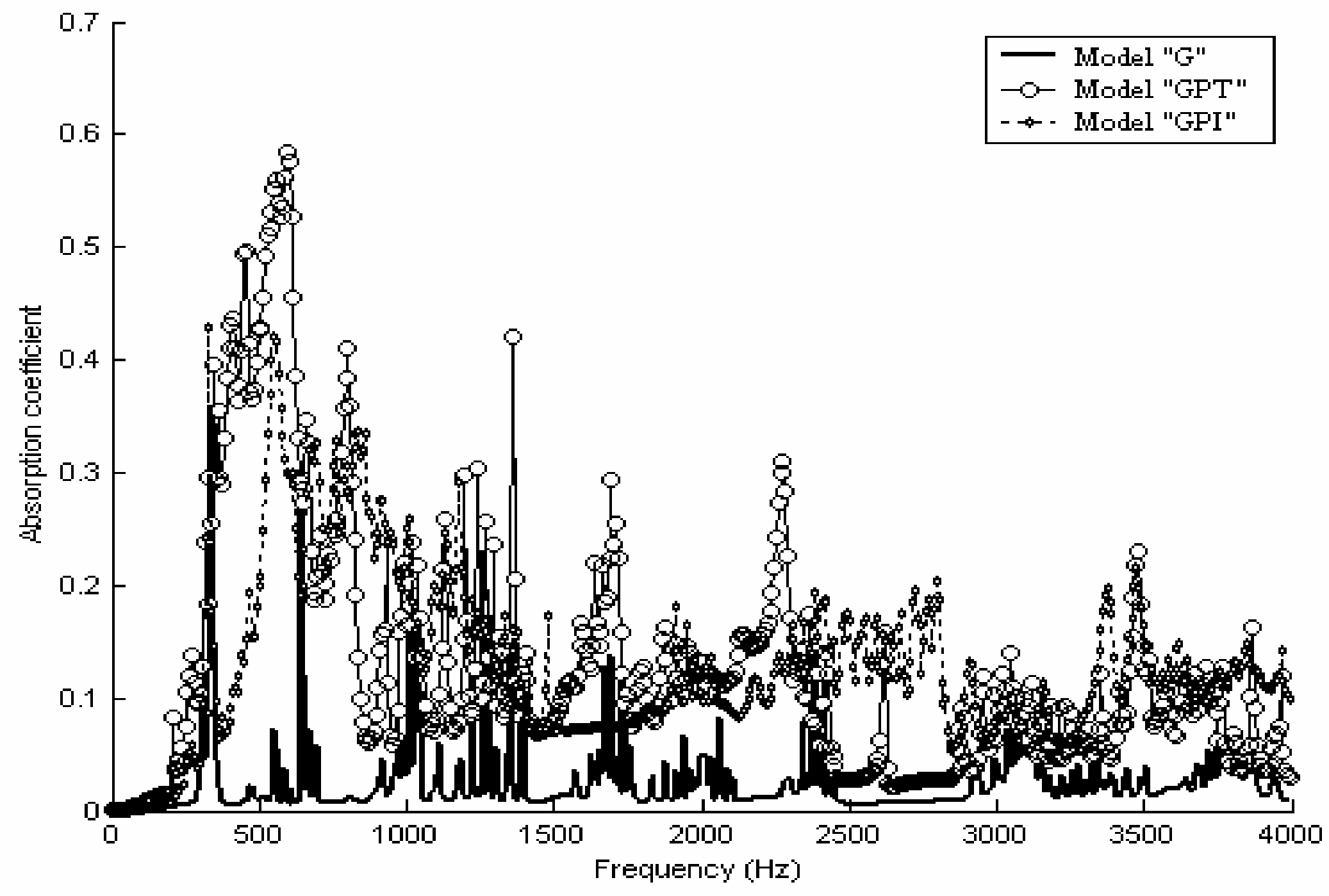




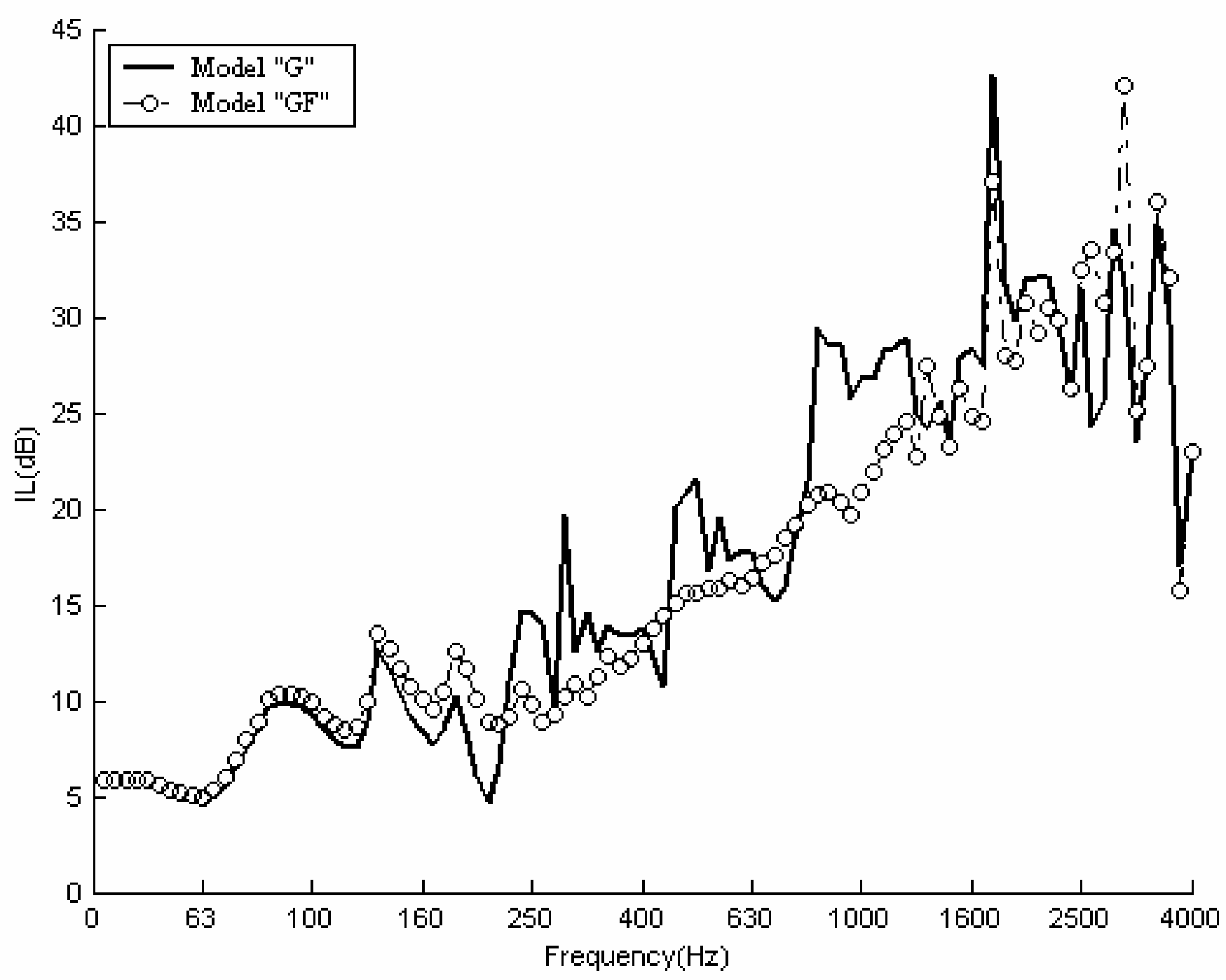




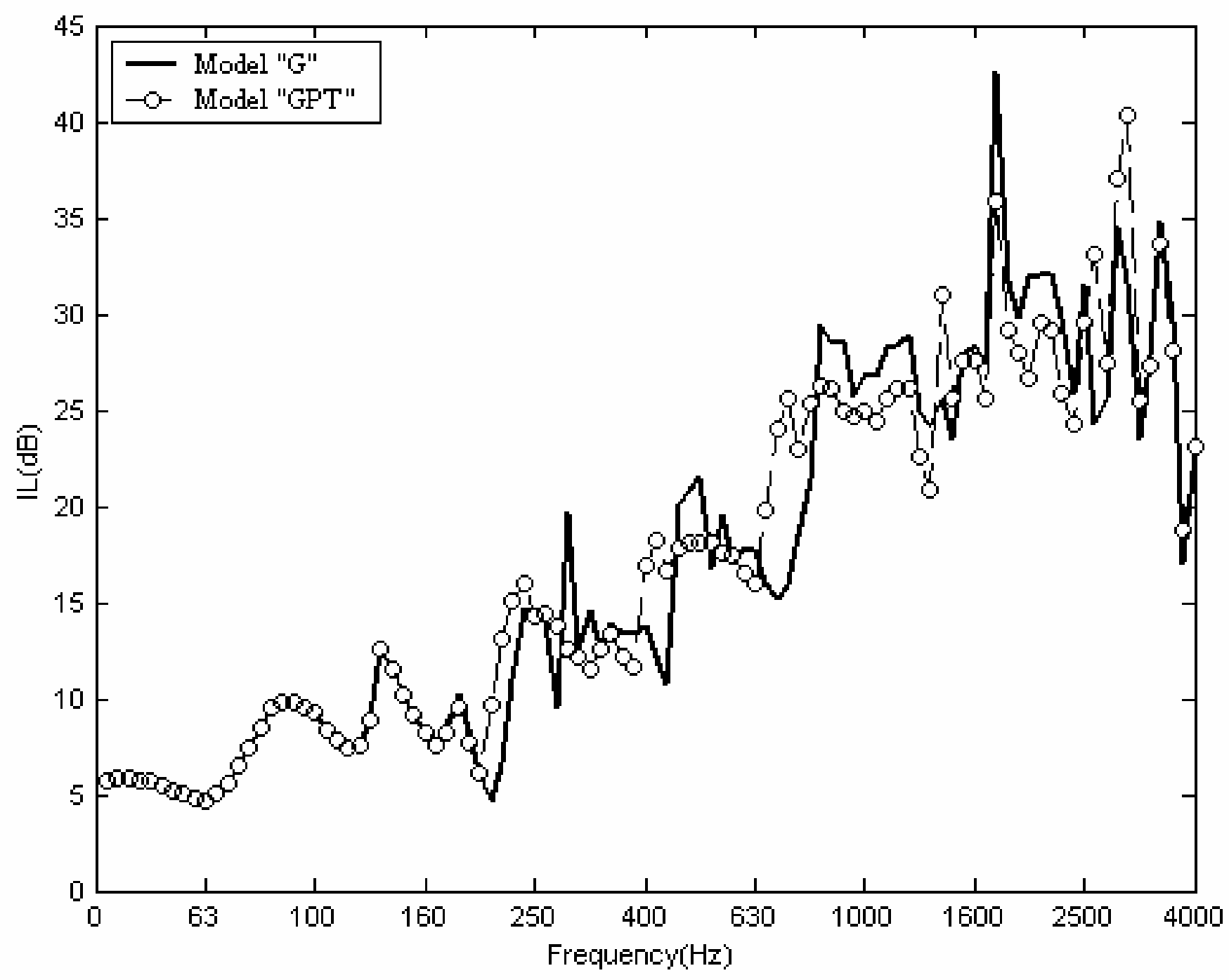




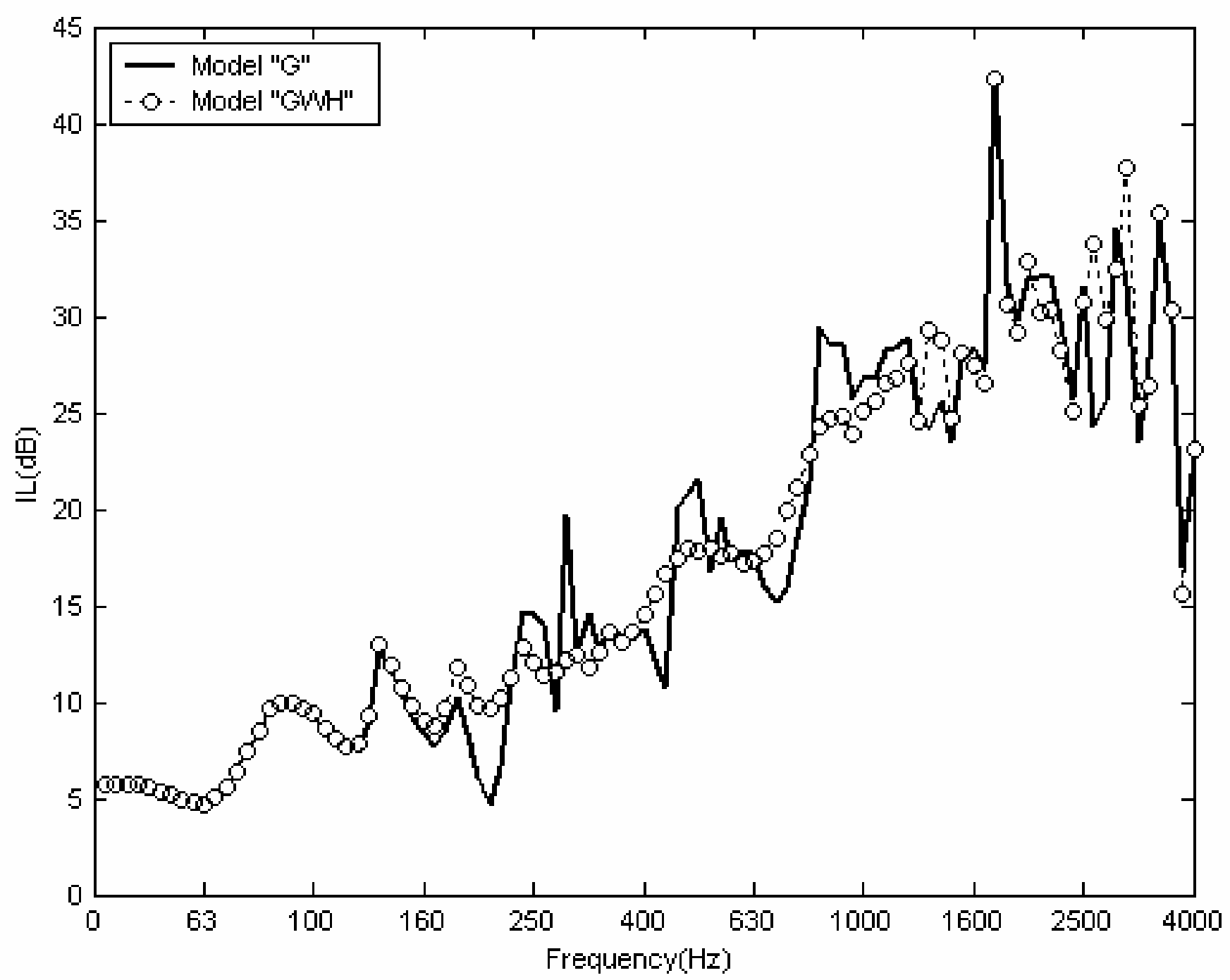




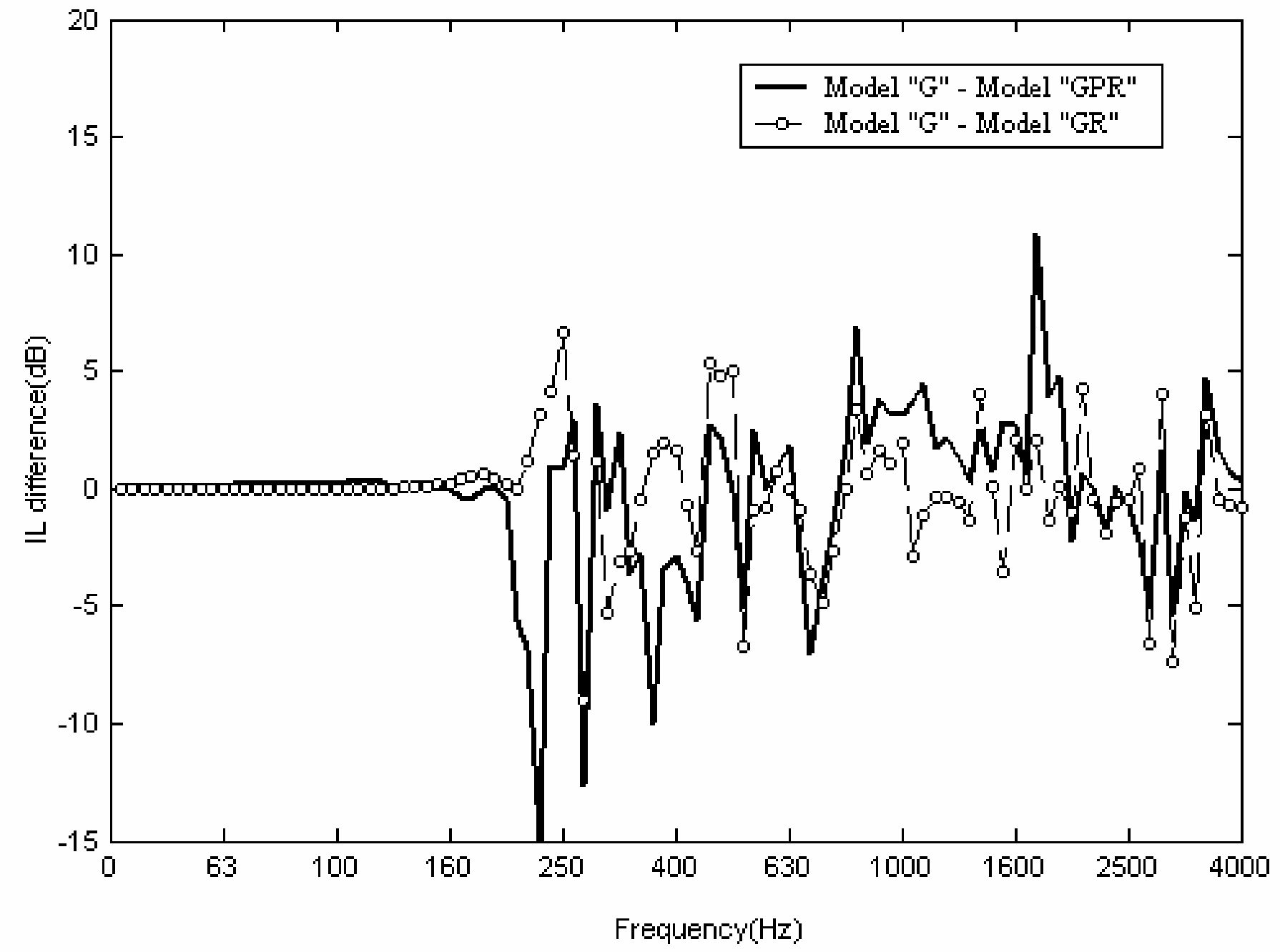




$$
-A A_{4}
$$




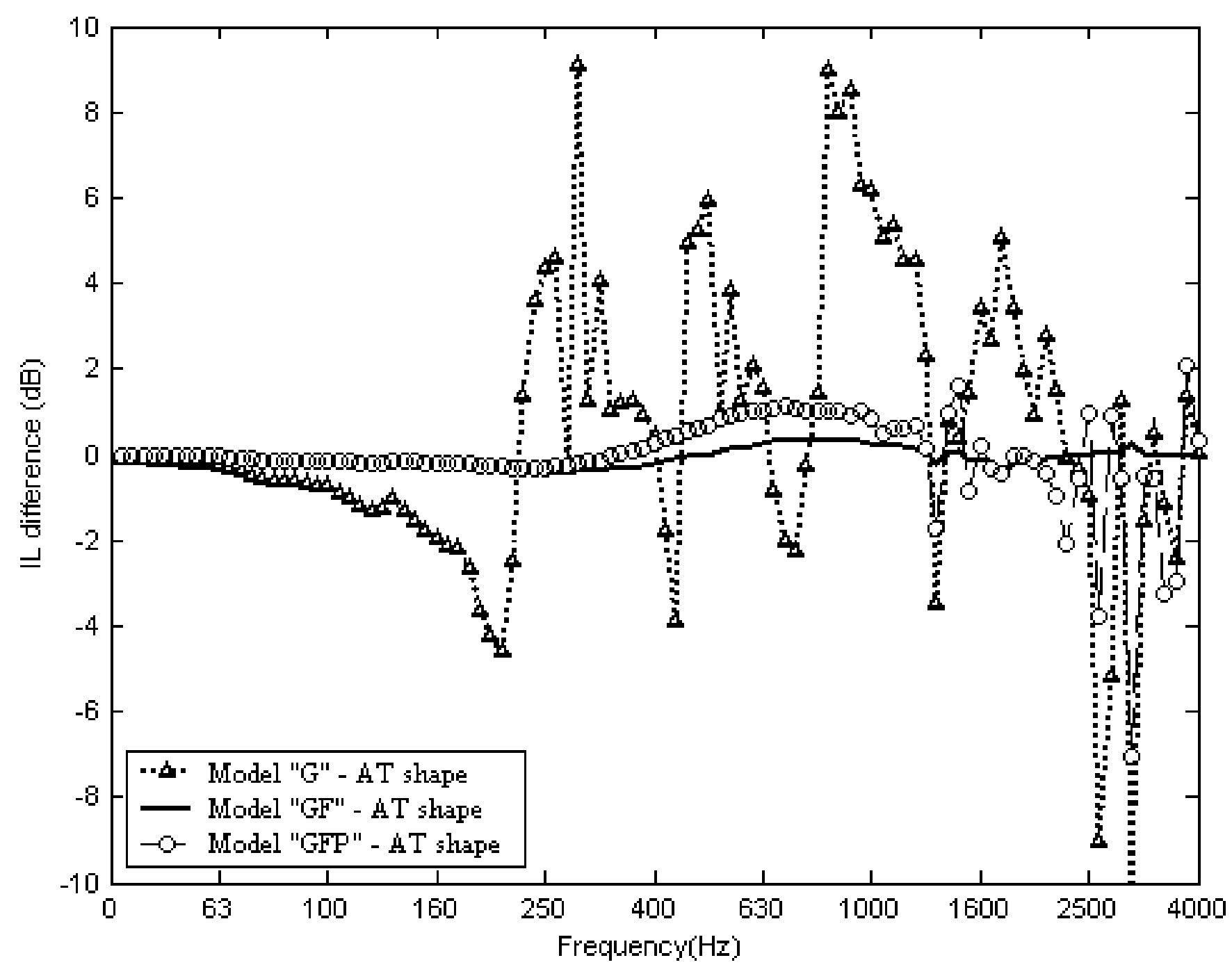




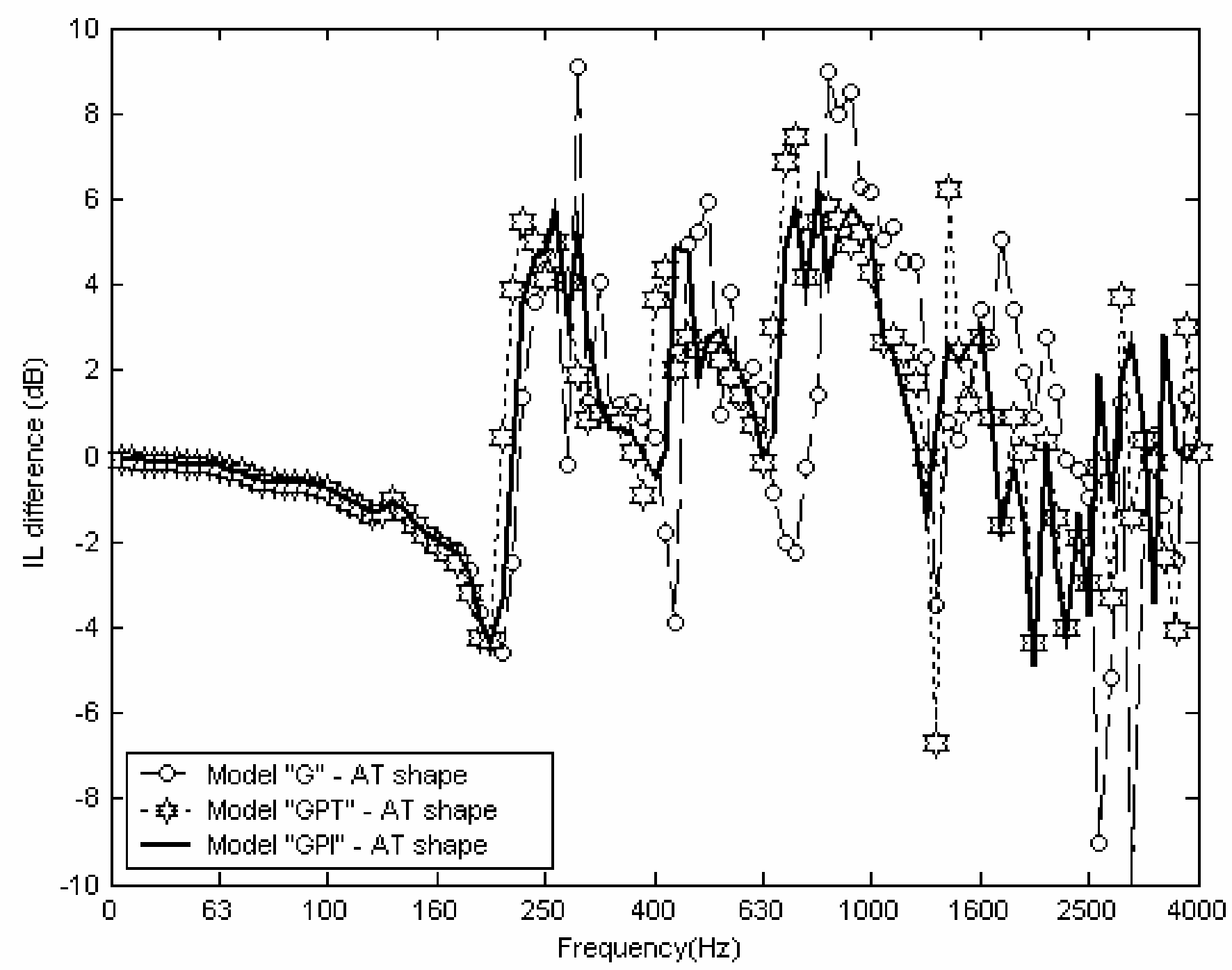




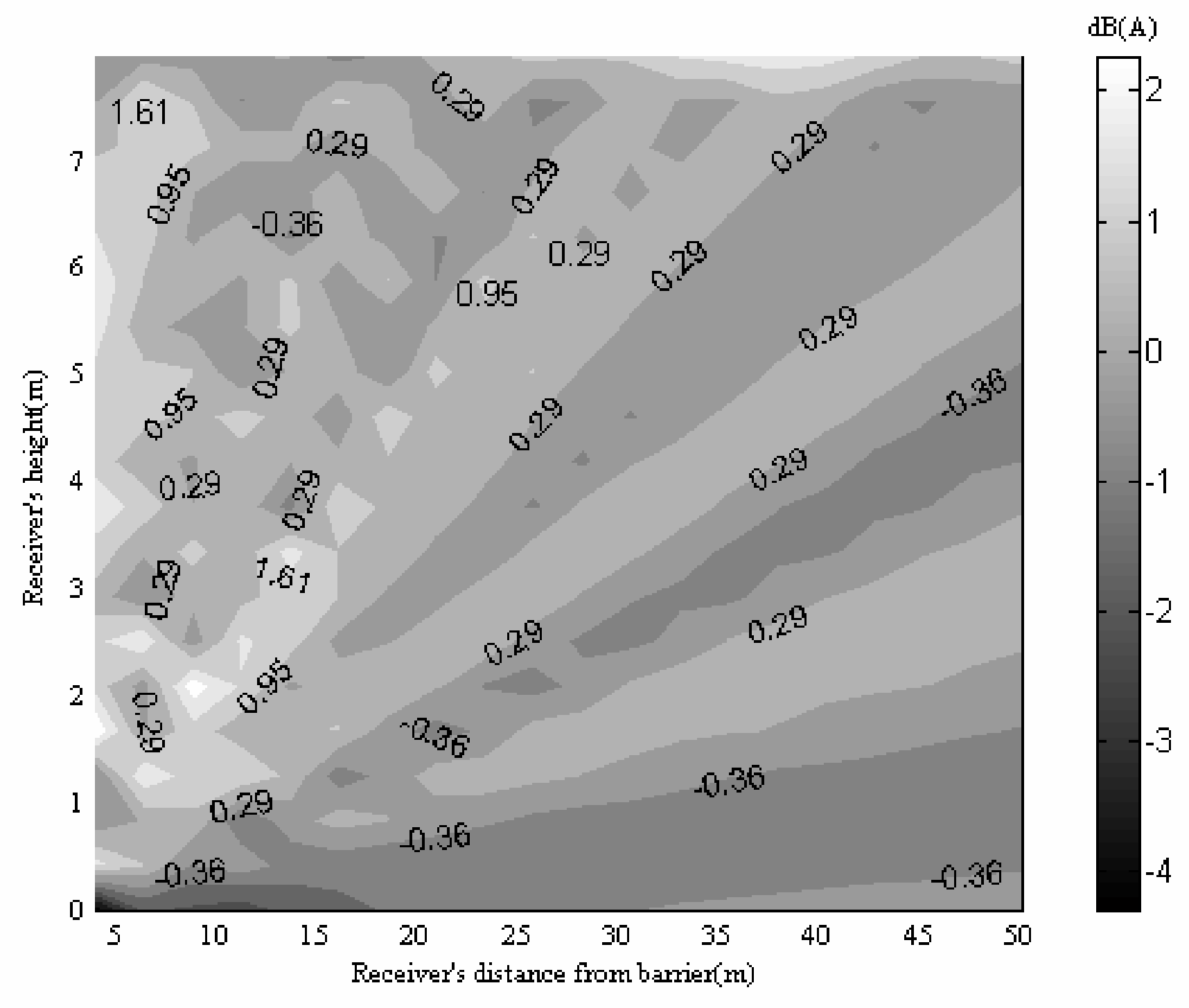


Rigid T shape barrier

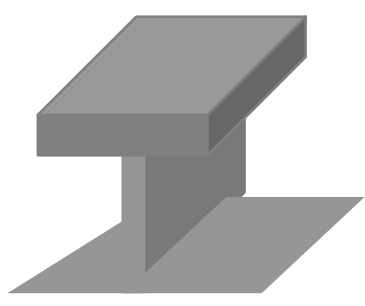

QRD barrier

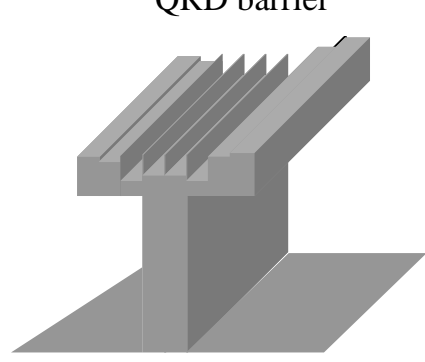

Stem: $(220 \times 30 \times 1.8 \mathrm{~cm})(\mathrm{L}, \mathrm{H}, \mathrm{W})^{*}$
Cap: $(220 \times 12.5 \times 15 \mathrm{~cm})(\mathrm{L}, \mathrm{H}, \mathrm{W})$

Overall height $=42.5 \mathrm{~cm}$

Source $(-1,0,0.08) \mathrm{m}$

Receiver $(2.5,0,0) \mathrm{m}$

Frequency Range $=200 \mathrm{~Hz}$ to $16 \mathrm{kHz}$

Stem: $(220 \times 30 \times 1.8 \mathrm{~cm})(\mathrm{L}, \mathrm{H}, \mathrm{W})$

Cap: $(220 \times 12.5 \times 15 \mathrm{~cm})(\mathrm{L}, \mathrm{H}, \mathrm{W})$

Overall height $=42.5 \mathrm{~cm}$

Frequency design $=1 \mathrm{kHz}$

Maximum well depth $=10 \mathrm{~cm}$

Well width $=1.8 \mathrm{~cm}$

Fin thickness $=0.32 \mathrm{~cm}$

Sequence (0 $10 \begin{array}{lllll} & 4 & 2 & 2 & 4\end{array}$ )

Source $(-1,0,0.08) \mathrm{m}$

Receiver $(2.5,0,0) \mathrm{m}$

Frequency Range $=200 \mathrm{~Hz}$ to $16 \mathrm{kHz}$ 

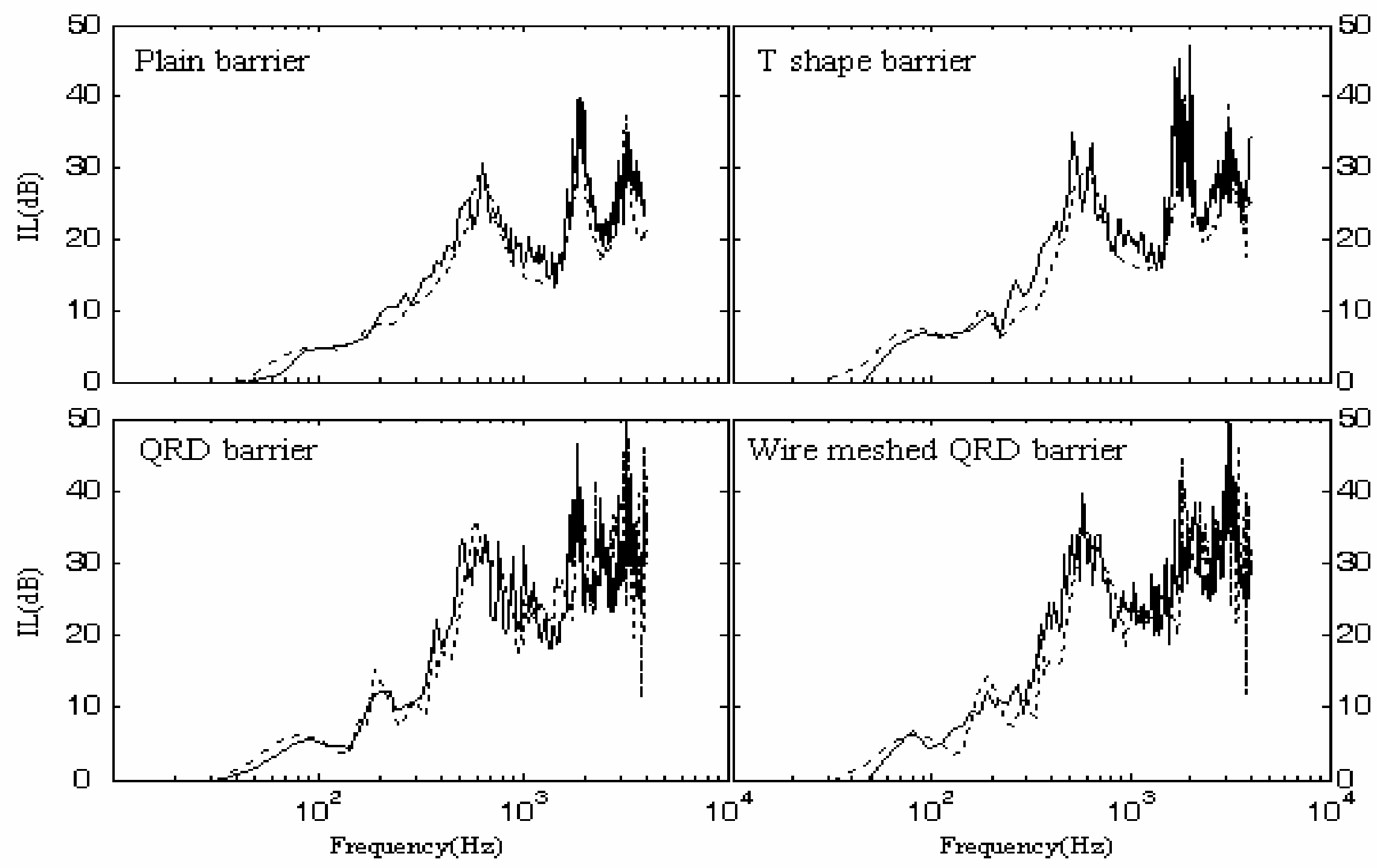